\title{
A Novel Group Paging Control Method for Massive MTC Accesses in LTE Networks
}

\section{Cong Wang ( $\nabla$ lgd_dolphin@139.com )}

Army Engineering University of PLA https://orcid.org/0000-0002-2731-7871

\section{Chengqiang Wei}

college of communications engineering, Army Engineering University of PLA

\section{Ning Li}

college of communications engineering,Army Egineering University of PLA

\section{Wenfeng Ma}

college of field engineering, Army Engineering University of PLA

\section{Hui Tian}

college of field engineering, Army Engineering University of PLA

\section{Research}

Keywords: 5G, Group paging, ACB, massive MTC devices (MTC), Machine to machine communication (M2M), overload control, random access

Posted Date: March 30th, 2020

DOl: https://doi.org/10.21203/rs.3.rs-15217/v2

License: (c) (i) This work is licensed under a Creative Commons Attribution 4.0 International License. Read Full License 


\title{
RESEARCH
}

\section{A Novel Group Paging Control Method for Massive MTC Accesses in LTE Networks}

Cong Wang ${ }^{1 *}$, Chengqiang $\mathrm{Wei}^{2}$, Ning $\mathrm{Li}^{2}$, Wenfeng $\mathrm{Ma}^{1}$ and Hui Tian ${ }^{1}$

\begin{abstract}
The fifth-generation (5G) systems have to deal with massive deployment of machine-type-communication (MTC) devices. System overload may occur during a random access (RA) procedure under a limited number of preamble resources and physical uplink shared channel $(\mathrm{PUSCH})$ resources especially when there exist massive MTC devices in a cell. In order to resolve the system overload (caused by the massive MTC deployment), the 3GPP proposed the adopted a group paging (GP)-based uplink access technique. But its performances dramatically decrease as the number of MTC devices increases. In this paper we propose a novel method, named ACB-based group paging overload control method (AGO). To reduce the number of simultaneous access MTC devices, AGO first scatters the MTC devices over a GP interval, and then automatically adjusts ACB parameters according to the load conditions. By doing so, AGO achieves high-channel access probability for MTC devices. Simulation results show that this method is superior to the GP and Pre-backoff (PBO) mechanisms in terms of success and collision probability, average access latency and resource utilization rate.
\end{abstract}

Keywords: 5G; Group paging; ACB; massive MTC devices (MTC); Machine to machine communication (M2M); overload control; random access

\section{Introduction}

The main goal of Internet of Things (IoT) is to ensure that massive machine type devices access the cellular networks. Machine-type Communication (MTC), also known as machine-to-machine (M2M) communication, is envisioned as a main enabler for the IoT [1]. According to its definition, MTC means the communications between machines (devices) without (or with a little) human intervention [2]. MTC applications have been experiencing rapid growth in various domains, such as intelligent transport systems, smart cities, eHealth, smart grids, industry automation, monitoring and control systems, etc. [3, 4].

With the development of IoT applications, the number of MTC devices is also growing rapidly. Unlike human-to-human $(\mathrm{H} 2 \mathrm{H})$ communications, in a cellular network, severe network congestion may occur when massive MTC devices initiate random access processes at the same time. Therefore, various methods have been proposed in the existing literature to solve the network congestion problem of the cellular systems. In general, these methods can be divided into two categories: pull-based methods and push-based methods.

\footnotetext{
${ }^{*}$ Correspondence: Igd_dolphin@139.com

${ }^{1}$ College of Field Engineering, Army Engineering University of PLA, 210007 Nanjing, China

Full list of author information is available at the end of the article
}

The access class barring (ACB) scheme and the group paging (GP) scheme are the more representative methods.

In Long-Term Evolution (LTE) networks, GP scheme is an effective mechanism recommended by the $3 \mathrm{GPP}$ group to resolve access conflicts. It is a very effective method to control network overload in the pull-based scheme [5]. In the GP scheme, user devices are grouped according to various quality of services (QoS), for example, delay-sensitive devices, delay-tolerant devices, time-controlled devices, mobile devices, fixed devices, etc. Each group is assigned an ID, called Group ID (GID). Therefore, user devices in a group can only be paged by one paging message. When receiving the paging message, all user devices of the group start a random-access procedure in the next available randomaccess slot at the same time. Since all devices in the same group initiate a random-access process at the same time, as the number of devices increases, the performance of the GP drops dramatically.

As a congestion control mechanism, $\mathrm{ACB}$ has been studied and used in 2G/3G networks [6]. In LTE/LTE$\mathrm{A}$, the ACB mechanism consists of an AC barring factor and an $\mathrm{AC}$ barring time parameter. These two parameters are broadcasted by the Evolved Node B (eNB) to the competing devices (including $\mathrm{H} 2 \mathrm{H}$ and 
M2M) through System Information Block (SIB). Before the device initiates the random-access process, it determines whether the group is prohibited according to the system information. Each device in the forbidden group chooses a random number between 0 and 1 , and then compares the random number to the ac barring factor. If it is smaller than the factor, the device starts a random-access process. Otherwise, the device will be force to enter the back-off process. Thus, MTC devices can achieve high access efficiency in ACB mechanism. However, it is difficult to set ACB parameters according the load dynamically.

In this paper we propose a novel method, named ACB-based group paging overload control method (AGO). To reduce the number of MTC devices simultaneously initiating a random-access process, AGO first scatters the MTC devices over a GP interval, and then automatically adjusts ac barring factor according to the load conditions.

The remainder of the paper is organized as follows: Section 2 gives a brief review of the related works. Section 3 introduces LTE Random Access Procedure. The AGO scheme is elaborated in section 4 . The performance evaluation of the AGO scheme is presented in section 5 . The paper is concluded in section 6 .

\section{Relate Works}

As massive MTC devices access the network, the problem of network overload becomes more and more serious. According to key problems in recent M2M communications, there are many studies being carried out to alleviate RAN overload and network congestion [7]. These studies divide control mechanism into pushbased and pull-based methods [5].

The pull-based mechanisms are central control methods. The network (eNB) initiates the RACH process, and only the paged MTC devices can access the network. As mentioned earlier, among all the pull-based mechanisms, the GP mechanism [5], recommended by the $3 \mathrm{GPP}$, is an effective mechanism for controlling network overload. In the GP mechanism, MTC devices are grouped into different groups, and only devices in the same group can be paged in the one paging message. Many literatures have studied the performance of the GP mechanism. Compared with the method of uniform distributing traffic, in the GP mechanism, all devices in the same group will simultaneously start a random-access process at the first time slot, which will result in an extremely high system load [8]. Therefore, some improvement methods of GP mechanism have been proposed. A method for controlling the random-access process of MTC devices through strict slot scheduling is proposed in [9]. A consecutive group paging (CGP) to enhance the performance of group paging is proposed in [10]. However, in some cases, the performance of CGP is worse than the classic GP mechanism. Further, an optimized GP mechanism, named FI-TSFGP, is proposed in [11]. Compared with the classical GP method, FI-TSFGP scatters the paging operation of the MTC devices over a GP interval. In this way, the FI-TSFGP mechanism has achieved high-channel access probability and reduced energy consumption at the same time. In addition, a method called Pre-backoff (PBO) has been proposed in $[12,13]$. In $\mathrm{PBO}$, some random-access requests are uniformly distributed over a pre-backoff window. PBO scheme can abate the number of MTC devices sending random access requests for a short time and thus can deal with the RAN congestion problem [12]. However, the size of the backoff window has a certain impact on the performance of the system. Then, some scholars started to explore how to set the size of the backoff window. For example, the author in [14] dynamically adjusts the backoff window to ensure that delayed devices do not enter the next paging cycle.

The push-based mechanisms are decentralized radio access network overload control methods. There are the following technologies: ACB mechanism, dynamic resource allocation mechanism and random-access resource separation mechanism, etc.

$\mathrm{ACB}$ mechanism is considered as the main solution for M2M communications access control. The existing literatures mainly focus on the dynamic adjustment method of ACB parameters [15, 16, 17, 18]. In [15], a dynamic adjustment of ACB parameters with PID is proposed. In order to adjust ACB parameters, trafficload needs to be estimated. In reference [16], according to the situation of network collision, a markov-chain based traffic-load estimation scheme is proposed. Further in $[17,18]$, two dynamic access class barring (DACB) algorithms have been presented. In the paper, these algorithms can adjust the ACB factors dynamical, and effectively improve system performance, such as reducing network delay and total system service time.

Unlike traditional $\mathrm{H} 2 \mathrm{H}$ communications, M2M communications service features are different, and the required QoS is also different. To meet different QoS requirements, authors in [19] propose a Multiple Access Class Barring (MACB) mechanism. In MACB, different access priorities are set for different applications. In order to improve access performance, 3GPP has provided the Extended Access Barring (EAB) mechanism. In EAB, the time-delayed MTC devices are divide into 0 to 9 regular access levels. The eNB can enable or restrict the random-access process of MTC devices with a certain access level according to the current overload condition [20]. Reference [21] proposed an EAB mechanism to enhance the performance of ACB scheme. 


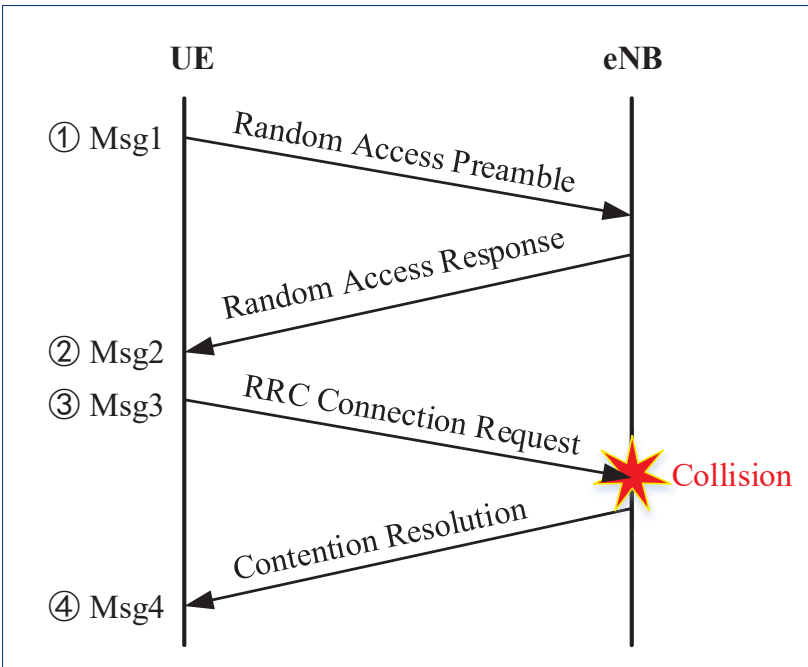

Figure 1 Random access process

Although the ACB and EAB mechanisms have a certain degree of containment against overload, they still cannot minimize the overload, and EAB mechanisms is mainly for time-tolerant business.

Both ACB and GP mechanism have disadvantages. As a push-based method, ACB is a decentralized approach, so, the overall resource utilization of the system is not stable. In addition, how to dynamically adjust ACB parameters according to load changes has always been a major problem. GP mechanism, as a pull-based methods, is a centralized control solution, and the overall resource utilization is stable. But GP method has some defects in signaling overhead and inflexibility. In addition, the ACB and GP mechanisms do not perform well in dealing with massive MTC device access.

\section{LTE Random Access Procedure}

In the LTE system, the user device can only perform data transmission after it has synchronized with the uplink of the system through a random-access process. Random access in LTE is divided into two forms: contention-based random access and contention-free random-access. This paper only studies contentionbased random-access methods. The random-access process in LTE system is shown in figure 1.

Random Access Preamble Transmission (Msg1): When a device starts an access request to the RACH, it will randomly select a preamble, and transmit the preamble to the eNB via PRACH. Since the preamble selection is randomly, if there are two or more devices selecting the same preamble, and these devices transmit the preamble to the eNB at the same time slot, then there will be a conflict.
Random Access Response (Msg2): After receiving the Msg1, eNB decodes the message, and transmit a RAR response message to the UE devices. The message includes a RA preamble identifier (ID), an uplink grant for MSG3, timing alignment (TA) command for corresponding UEs, and assignment of a temporary identifier (the cell radio network temporary identifier, CRNTI). UEs expect to receive RAR messages within a certain time. If a device doesn't receive RAR message during the certain time, the back-off mechanism will be activated in the back-off window $\left(W_{B O}\right)$.

RRC Connection Request (Msg3): When the UE receives the RAR response message, it will send a connection request message with the assigned resource block (RB) on PUSCH to the eNB. The devices, with a preamble conflict in step 1 , are configured the same RBs. So, the eNB cannot decode the message of these devices. This will cause the random-access process to fail.

Contention resolution (Msg4): The eNB receives the $\mathrm{RRC}$ connection request message. If the eNB can decode the message successfully, it will transmit a contention resolution message to the related devices. If a device receives the contention resolution message, it means that the random-access procedure was successful. Otherwise, it will perform a fallback operation, and launch a new access request later.

\section{ACB Based Group Paging Overload Control Method (AGO)}

4.1 System Model

We consider a single cell LTE network consisting of a single eNB and a number of MTC devices $(M)$. We assume that these devices are divided into $N$ groups, and are uniformly distributed over the groups. Therefore, there are $(M / N)$ MTC devices in a group. The system model is showed in Figure 2. The eNB reserves $R$ channel resources for contention-based random-access. In this way, the total available resources for contentionbased random-access are equal to the number of frequency bands in a random-access slot multiplied by the number of preambles. For the sake of analysis, we assume that there is only one available frequency band in each random time slot. Therefore, the total available random-access resources are equal to the number of available preambles.

In classical GP method, when the devices in the GID group receive the paging message, which is addressed by the GID, they will initiate the randomaccess quest at the same time. Different from the classic GP method, AGO combines the ACB mechanism and GP mechanism, and performs ACB mechanism in the paged group. Only those devices that pass ACB detection can initiate a contention-based random-access procedure. 


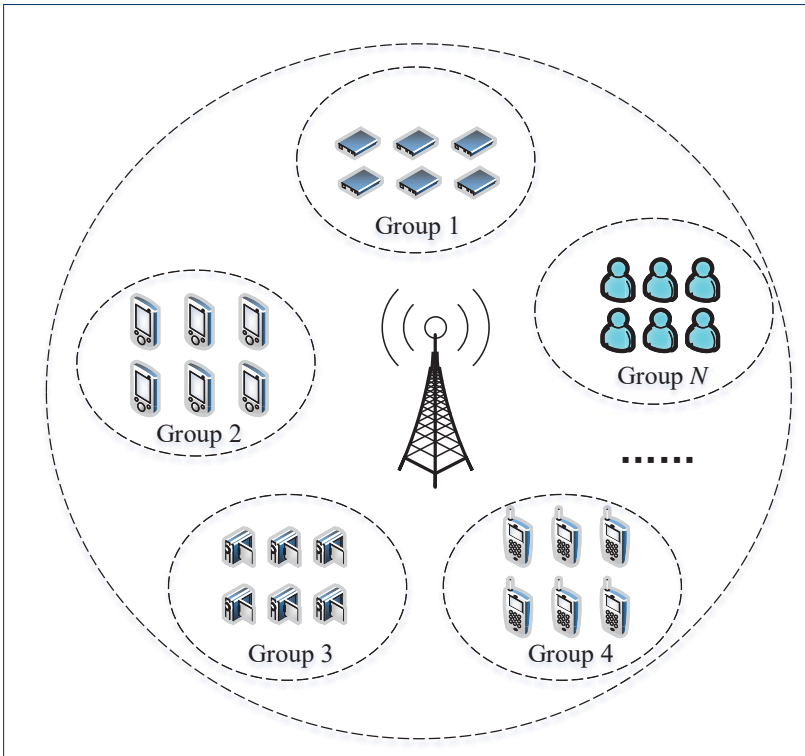

Figure 2 System model

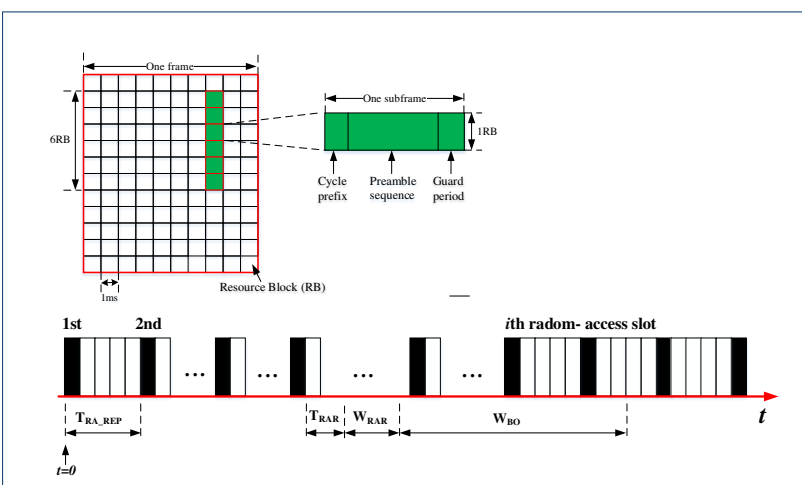

Figure 3 Random access time slot and resource allocation

\subsection{Group Paging Mechanism}

In the LTE system, time is divided into fixed-length radio frames. As shown in Figure 3, there are several radio subframes in each radio frame. Random-access slot refers to the radio subframe (one or more) used by the devices to complete the random-access process.

In the initial state, after receiving the paging message, the MTC devices in the corresponding group will send the 1st preambles in the first random access slot. If the MTC device competition failed, they will perform a fallback process, wait for a period of time, and then initiate a random-access request again. We assume that $N_{P T_{\max }}$ represents the maximum number of times the device can retransmit preambles during the paging interval. Then the device re-initiates a randomaccess request until it successfully accesses or the number of retransmissions reaches $\left(N_{P T_{\max }}-1\right)$ times. For the convenience of discussion, we define the following symbols: $T_{R A R}$ represents the processing delay of the base station, $T_{R A} \_$REP represents the interval between two consecutive RA slots, $W_{B O}$ represents the size of the backoff window, $W_{R A R}$ represents the number of random-access response messages (RAR) contained in the random-access response window, and $N_{R A R}$ represents the maximum number of RARs per a response message.

After sending the Msg1(Random Access Preamble Transmission Message), the MTC device waits to receive RAR message within $T_{R A R}+W_{R A R}$ subframes. We can get the number of RARs included in the RA window as follows:

$$
N_{A C K}=N_{R A R} \times W_{R A R}
$$

During each preamble transmission, MTC devices need to wait for $\left(T_{R A R}+W_{R A R}+W_{B O}\right)$ radio subframes before resending a new preamble. We can get the maximum number of RA slots for group paging $\left(I_{\max }\right)[22]:$

$$
I_{\max }=1+\left(N_{P T_{\max }}-1\right)\left\lceil\frac{T_{R A R}+W_{R A R}+W_{B O}}{T_{R A \_R E P}}\right\rceil
$$

Let $R$ represents the number of preambles reserved by the eNB in each RA slot and $p_{1}$ be the detection probability for the first preamble transmission. Then the number of MTC devices that have successfully transmitted the 1st preamble in RA slot is equal to:

$$
M_{s}=M e^{-\frac{M}{R}} p_{1}
$$

Where $M$ represents the total number of MTC devices that will transmit the 1st preamble. If the device fails to transmit the preamble, it will back off for a certain time and then send the preamble again until it reaches the maximum number of retransmissions. Let $p_{n}$ be the detection probability for the $n^{\text {th }}$ preamble transmission. And then we can get the number of MTC devices that have successfully transmitted the $n^{\text {th }}$ preamble in RA slot:

$$
M_{s}[n]=M[n] e^{-\frac{M[n]}{R}} p_{n}
$$

Where $M[n]$ represents the total number of MTC devices that will transmit the $n^{\text {th }}$ preamble and $p_{n}=1-$ $e^{-n}$. 
Since we divide the paging interval into $I_{\max }$ different RA time slots. In this way, at each RA slot, the number of devices that initiate access requests is equal to the total number of devices that send $n^{\text {th }}$ preambles. Here $n^{\text {th }}$ is equal to $1,2,3, \ldots, N_{P T_{\max }}$.

$M_{i}$ represents the number of MTC devices that started the RA procedure in the $i$ slot. $M_{i}[n]$ represents the number of MTC devices that transmitted the preamble for the $n^{t h}$ times in the $i^{t h}$ slot. $M_{i, s}[n]$ is the number of devices that successfully transmitted the preamble for the $n^{t h}$ times in $\operatorname{slot}(i) . M_{i, c}[n]$ is the number of devices that failed to transmit the preamble for the $n^{\text {th }}$ times in $\operatorname{slot}(i)$. The number of successful MTC devices is equal to [22]:

$$
M_{i, s}[n]= \begin{cases}M_{i}[n] e^{-\frac{M_{i}}{R}} p_{n}, & \text { if } M_{i, s} \leq N_{A C K} \\ \frac{M_{i}[n] e^{-\frac{M_{i}}{R}} p_{n}}{M_{i, s}} N_{A C K}, & \text { otherwise }\end{cases}
$$

$$
\begin{aligned}
& M_{i, c}[\mathrm{n}]=\mathrm{M}_{i}[n]-M_{i, s}[\mathrm{n}] \\
& = \begin{cases}\mathrm{M}_{i}[n]\left(1-e^{-\frac{M_{i}}{R}} p_{n}\right), & \text { if } M_{i, s} \leq N_{A C K} \\
\mathrm{M}_{i}[n]\left(1-\frac{p_{n}}{M_{i, s}}\right) N_{A C K}, & \text { otherwise }\end{cases}
\end{aligned}
$$

Let $M_{i, s}$ be the number of MTC devices that have successfully transmitted the preamble and finished the contention-based RACH procedure at RA slot $(i)$. It can be defined as follows.

$$
M_{i, s}=\sum_{n=1}^{N_{P T_{\max }}} M_{i}[n] e^{-\frac{M_{i}}{R}} p_{n}
$$

Then, $M_{i}$ can be derived by the following equation [23]:

$$
M_{i}=\sum_{n=1}^{N_{P T_{\max }}} M_{i}[n]
$$

\subsection{ACB mechanism}

If there are a large number of devices waiting for access in RA time slot $(i)$, access conflicts will still be caused, resulting in network performance degradation. In order to reduce the collision probability, AGO dynamically adjusts the ACB parameters according to the amount of loading in each RA slot.

$\mathrm{AC}$ barring factor is the most important parameter in the ACB mechanism. It is a number between 0 and 1, indicating the probability of forbidden access to the network. If an MTC device belongs to a forbidden group, its ability to initiate a random-access process depends on the ac barring factor. The device randomly selects a value between $[0,1]$ and compares it with the value of ac barring factor. If the value is less than the factor, the MTC device can initiate the random-access process, otherwise it is barred [24].

Let $P\left(M_{i, s}=m \mid M_{i}=n\right)$ be the probability of $m$ devices successfully completing the random access process, while $n$ devices attempt to access the network. The probability $P$ consists of two parts:

- Probability of $k$ out of $n$ devices passing through ACB mechanism, $P\left(M_{A C B}^{i}=k \mid M_{i}=n\right)$.

- In the case where $k$ devices pass ACB detection, the probability of $m$ devices completing the random access process, $P\left(M_{i, s}=m \mid M_{A C B}^{i}=k\right)$.

So, $P\left(M_{i, s}=m \mid M_{i}=n\right)$ is equal to:

$$
\begin{aligned}
& P\left(M_{i, s}=m \mid M_{i}=n\right)= \\
& \sum_{k=0}^{n} P\left(M_{A C B}^{i}=k \mid M_{i}=n\right) \times P\left(M_{i, s}=m \mid M_{A C B}^{i}=k\right)
\end{aligned}
$$

Let $p_{a c b}$ be the ac barring factor, and then we can get:

$$
\begin{aligned}
& P\left(M_{A C B}^{i}=k \mid M_{i}=n\right)= \\
& \left(\begin{array}{l}
n \\
k
\end{array}\right) p_{a c b}{ }^{k}\left(1-p_{a c b}\right)^{n-k}, 0 \leq k \leq n
\end{aligned}
$$

Among the $R$ preambles, the probability of each preamble being selected is the same, which is equal to $1 / R$. In this case, the probability of any device that completes the random access process is [25]:

$$
P\left(Z_{j}=1 \mid M_{A C B}^{i}=k\right)=\left(\begin{array}{c}
k \\
1
\end{array}\right) \frac{1}{R}\left(1-\frac{1}{R}\right)^{k-1}
$$

Where $Z_{j}$ represents the state of the preamble $j$. $Z_{j}=1$ means that the current preamble $j$ is selected by only one device, $Z_{j}=0$ means that the current preamble $j$ is not selected, and $Z_{j}=c$ means that the current preamble $j$ is selected by two or more devices.

So, we can get the expected value of the number of preambles successfully transmitted, and it can be defined as: 


$$
E\left(M_{i, s} \mid M_{A C B}^{i}=k\right)=\sum_{j=1}^{R}\left(\begin{array}{l}
k \\
1
\end{array}\right) \frac{1}{R}\left(1-\frac{1}{R}\right)^{k-1}
$$

Combining formulas 9,10 and 12, in the random access slot $i$, when the total number of devices to be accessed is $n$, the expected value of the number of successfully transmitted preambles is:

$$
\begin{aligned}
& E\left(M_{i, s} \mid M_{i}=n\right) \\
& =\sum_{k=1}^{n}\left(\begin{array}{l}
n \\
k
\end{array}\right) p_{a c b}{ }^{k}\left(1-p_{a c b}\right)^{n-k}\left(\begin{array}{c}
k \\
1
\end{array}\right)\left(1-\frac{1}{R}\right)^{k-1} \\
& =n p_{a c b}\left(1-\frac{p_{a c b}}{R}\right)^{n-1}
\end{aligned}
$$

Therefore, the probability that the preamble selected by any device is successfully transmitted is:

$$
P_{S}=p_{a c b}\left(1-\frac{p_{a c b}}{R}\right)^{n-1}
$$

Differentiating Equation 14 with respect to $p_{a c b}$, we can get:

$$
\frac{\mathrm{d} P_{S}}{\mathrm{~d} p_{a c b}}=\left(1-\frac{p_{a c b}}{R}\right)^{n-2}\left(1-\frac{n p_{a c b}}{R}\right)
$$

According to formula 15, we can draw the following conclusions:

- In the case of $R>=n$, if $p_{a c b}=1$, the maximum access success probability can be obtained.

- In the case of $R<n$, if $p_{a c b}=R / n$, we can get the maximum access success probability.

In summary, we can know that the optimal ac barring factor is [26]:

$$
p_{a c b}^{*}=\min \left(1, \frac{R}{M_{i}}\right)
$$

When the random-access process occurs, the eNB calculates the optimal ac barring factor according to the network load in the current time slot. As the author did in the reference [27], we can use the idle or the collision preambles to estimate network load. Devices attempting to access the eNB randomly select a value between $[0,1]$ and compare it with the ac barring factor. Only devices with random number less than the value of $\mathrm{ACB}$ parameter may be allowed to participate in the random-access process. Devices that fail the ACB test will start the back-off mechanism and wait for the next access opportunity.

Finally, the number of competing devices in each random-access slot can be dynamically controlled by equation 17.

$$
M_{i}=\sum_{n=1}^{N_{P T_{\max }}}\left(M_{i}[n] \times p_{a c b}^{*}\right)
$$

\subsection{Analytical Model}

In this section, we will use an analytical model [11] to analyze the performance of the AGO.

At the first available RA slot, the devices, which are paged, will send the preamble. Due to the limited number of preamble resources and physical uplink shared channel (PUSCH) resources, some device will be collided. Through the previous analysis, we know that the number of devices that send the preamble for the first time in each RA slot should be equal to the number of newly arrived devices, for example, $M_{i}[1]=M_{a r v}$. Then after the first time slot is completed, the number of successfully connected and failed devices are [11]:

$$
\begin{aligned}
M_{1, s} & = \begin{cases}M_{i} e^{-\frac{M_{i}}{R}} p_{1}, & \text { if } M_{i} e^{-\frac{M_{i}}{R}} p_{1} \leq N_{A C K} \\
N_{A C K}, & \text { otherwise }\end{cases} \\
M_{1, c} & =M_{1}-M_{1, s}
\end{aligned}
$$

After the RAR window, the device that fails to transmit the preamble at the first time will go back for a certain time and restart the random-access process. Since the back-off time is uniformly distributed, the collided devices are uniformly distributed over the back-off window. The number of devices that transmit the preamble at the second time in a RA slot is equal to the part of slots (named as $\partial_{a}, \partial_{b c}$ and $\partial_{d}$ ), from the backoff interval, falling before this RA slot multiplied by the number of collided MTC devices. The position of the first RA slot $(a)$ in the back-off window is shown in Figure 4. The figure shows that the first preamble transmission process and the devices that failed to access the eNB follow the back-off mechanism and wait for the second preamble transmission. From the figure, the position of the first RA slot $(a)$ in the back-off window is as follows [11]:

$$
x_{a}(i)=i+\left\lceil\frac{T_{R A R}+W_{R A R}}{T_{R A \_R E P}}\right\rceil
$$


Where, $x_{a}(i)$ is the order of the first RA slot in the back-off window. The proportion of the MTC devices for the second preamble transmission in time slot $(a)$ is equal to the time of the slot $(a)$, in a subframe unit, minus the duration before the start of the back-off window (normalized by $W_{B O}$ ):

$$
\partial_{a}=\frac{\left\lceil\frac{T_{R A R}+W_{R A R}}{T_{R A \_R E P}}\right] T_{R A \_R E P}-\left(T_{R A R}+W_{R A R}\right)}{W_{B O}}
$$

Regarding the RA slots from (b) to (c), they will be just after the RA slot $(a)$, i.e.:

$$
x_{b c}(i)=i+\left\lceil\frac{T_{R A R}+W_{R A R}}{T_{R A \_R E P}}\right\rceil+h
$$

where, $h=1,2, \ldots, H_{\max } \cdot H_{\max }$ represents the number of RA slots between the slots $(b)$ and $(c)$. It is equal to $H_{\max }=\left\lfloor\left(W_{B O}-\partial_{a} W_{B O}\right) / T_{R A \_R E P}\right\rfloor$. However, the proportion of MTC devices that retransmit their preambles at these RA slots is equal to:

$$
\partial_{b c}=\frac{T_{R A \_R E P}}{W_{B O}}
$$

The remaining devices will be retransmitted in the RA slot $(d)$, then the position of RA slot $(d)$ :

$$
x_{d}(i)=i+\left\lfloor\frac{T_{R A R}+W_{R A R}+W_{B O}}{T_{R A \_R E P}}\right\rfloor+1
$$

The proportion of devices that retransmit in RA slot $(d)$ :

$$
\begin{aligned}
& \partial_{d}=1-\partial_{a}-\partial_{b c} H_{\max } \\
& =\frac{T_{R A R}+W_{R A R}+W_{B O}}{W_{B O}}- \\
& \frac{T_{R A \_R E P}}{W_{B O}}\left\lfloor\frac{T_{R A R}+W_{R A R}+W_{B O}}{T_{R A \_R E P}}\right\rfloor
\end{aligned}
$$

Since more than $N_{A C K}$ devices cannot get network services, formula 5 can be rewritten as:

$$
M_{i, s}[n]=M_{i}[n] e^{-\frac{M_{i}}{R}} p_{n}
$$

Therefore, we can get the following results:

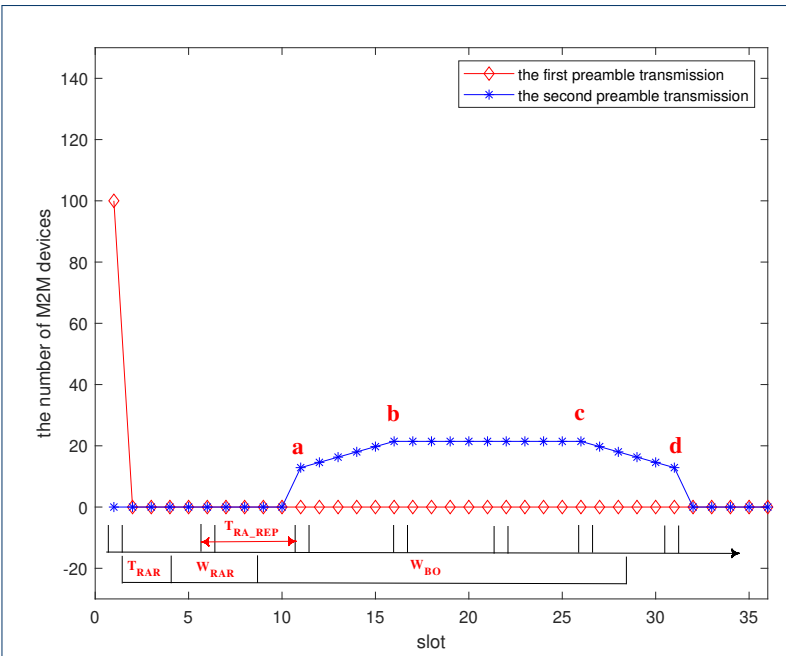

Figure 4 Number of MTC devices at each RA slot for the first and second preamble transmission for $R=54$ and $M / N=100[11]$

$$
M_{i, s}[1]=M_{i}[1] e^{-\frac{M_{i}}{R}} p_{1}
$$

$$
M_{i, c}[1]=M_{i}[1]-M_{i, s}[1]=M_{i}[1]\left(1-e^{-\frac{M_{i}}{R}} p_{1}\right)
$$

According to equation 8 , when $t=1$, we have:

$$
M_{1}=\sum_{n=1}^{N_{P T_{\max }}} M_{1}[n]
$$

It is easy to get from Figure 4:

$$
\begin{aligned}
& M_{1}[1]=M_{a r v} \\
& M_{1}[2]=M_{1}[3]=\cdots=M_{1}\left[N_{P T_{\max }}\right]=0 \\
& M_{1}=M_{a r v} \\
& M_{1, c}[1]=M_{1}[1]\left(1-e^{-\frac{M_{1}}{R}} p_{1}\right)
\end{aligned}
$$

Similarly, we can get $M_{2}$, and $M_{2}=M_{a r v}$.

Continually, when $t=3$, we can get:

$$
M_{3}=\sum_{n=1}^{N_{P T_{\max }}} M_{3}[n]
$$




$$
\begin{aligned}
& M_{3}[1]=M_{a r v} \\
& M_{3}[2]=\partial_{a} M_{1, c}[1]=\partial_{a} M_{1}[1]\left(1-e^{-\frac{M_{i}}{R}} p_{1}\right) \\
& M_{3}[3]=M_{3}[4]=\cdots=M_{3}\left[N_{P T_{\max }}\right]=0 \\
& M_{3}=M_{\text {arv }}+\partial_{a} M_{1}[1]\left(1-e^{-\frac{M_{1}}{R}} p_{1}\right)
\end{aligned}
$$

By induction, we can get $M_{i}$, for each time $\operatorname{slot}(i)$.

In the previous hypothesis, we considered that the newly arrived devices for each time slot is constant, $M_{\text {arv }}$. But in practical systems, in general, the device arrival rate obeys a specific distribution. For the burst massive MTC devices access scenario, we adopt the beta distribution as the MTC arrival model, and its distribution period is $I_{\max }$. As in reference [28], the parameters of beta distribution are $\alpha=3$ and $\beta=4$.

$$
g(t)=\frac{t^{\alpha-1}\left(I_{\max }-t\right)^{\beta-1}}{I_{\max }^{\alpha+\beta-1} B(\alpha, \beta)}
$$

$t$ represents the sequence number of RA slot. $B(\alpha, \beta)$ is the Beta function, and $g(t)$ is the distribution probability of M2M devices at each random access time. Therefore, the number of new arrivals of MTC devices in each random-access slot is $M_{\text {arv }}(t)$ :

$$
M_{\text {arv }}(t)=(M / N) \int_{t}^{t+1} g(t) d t
$$

Assuming $M / N=1000$, The number of total devices, the successful devices and the collision devices for each preamble transmission in each random-access slot are shown in Figure 5.

The change rule of the ac barring factor and the number of MTC devices under different conditions are shown in Figure 6. When $M / N=500$, the number of devices in each time slot is less than the number of preamble available, and then the ACB mechanism does not work, so $M_{i}[1]=M_{a r v}(i)$. When the number of devices in each time slot is greater than the number of preamble available, such as $M / N=2500$ or $M / N=$ 5000 , the ACB mechanism begins to work to prevent too many devices from initiating access requests in the same time slot. As shown in the Figure 6, the number of devices is greatly reduced.

\section{Performance Evaluation}

\subsection{Performance Metrics}

We investigate five performance metrics to evaluate the proposed AGO method: Average access delay, success probability, collision probability, the times of

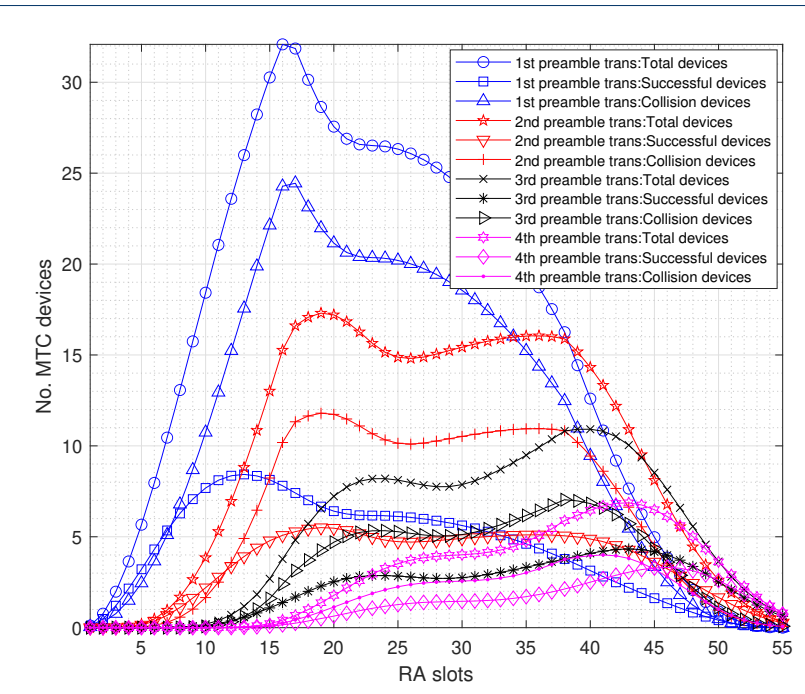

Figure 5 The number of the total devices, the successful devices and the collision devices for each preamble transmission in each random-access slot; $M / N=1000$ , $P T_{\max }=10, \alpha=3, \beta=4$.

preamble transmission, and resource utilization. In order to show the performance of AGO, AGO will be compared with two classic methods: GP and PBO $\left(W_{P B O}=100\right.$ and $W_{P B O}=240$, respectively $)$. The simulation parameters are shown in Table 1.

\section{A. Average Delay}

The Average Delay $\left(\overline{D_{a}}\right)$ represents the average time for the device to finish the random access process successfully. It is equal to the total time delay of the devices that successfully completed the access process divided by the number of devices that completed the access process successfully. $T_{i}$ represents the time from the device initiating the request to completing the preamble and information transmission in RA slot $i$. The definition of $T_{i}$ is as follows [22]:

$$
T_{i}=(i-1) T_{R A \_R E P}+\left(T_{R A R}+W_{R A R}\right)+\overline{T_{M S G}}
$$

$\overline{T_{M S G}}$ represents the average time for the device to successfully transmit information (including message 3 and message4). In this paper, we assume that $\overline{T_{M S G}}$ is $11 \mathrm{~ms}$, so the average access delay is:

$$
\overline{D_{a}}=\frac{\sum_{i=1}^{I_{\max }} \sum_{n=1}^{N_{P T_{\max }}} M_{i, s}[n] T_{i}}{\sum_{i=1}^{I_{\max }} \sum_{n=1}^{N_{P T_{\max }}} M_{i, s}[n]}
$$

\section{B. Success Probability}




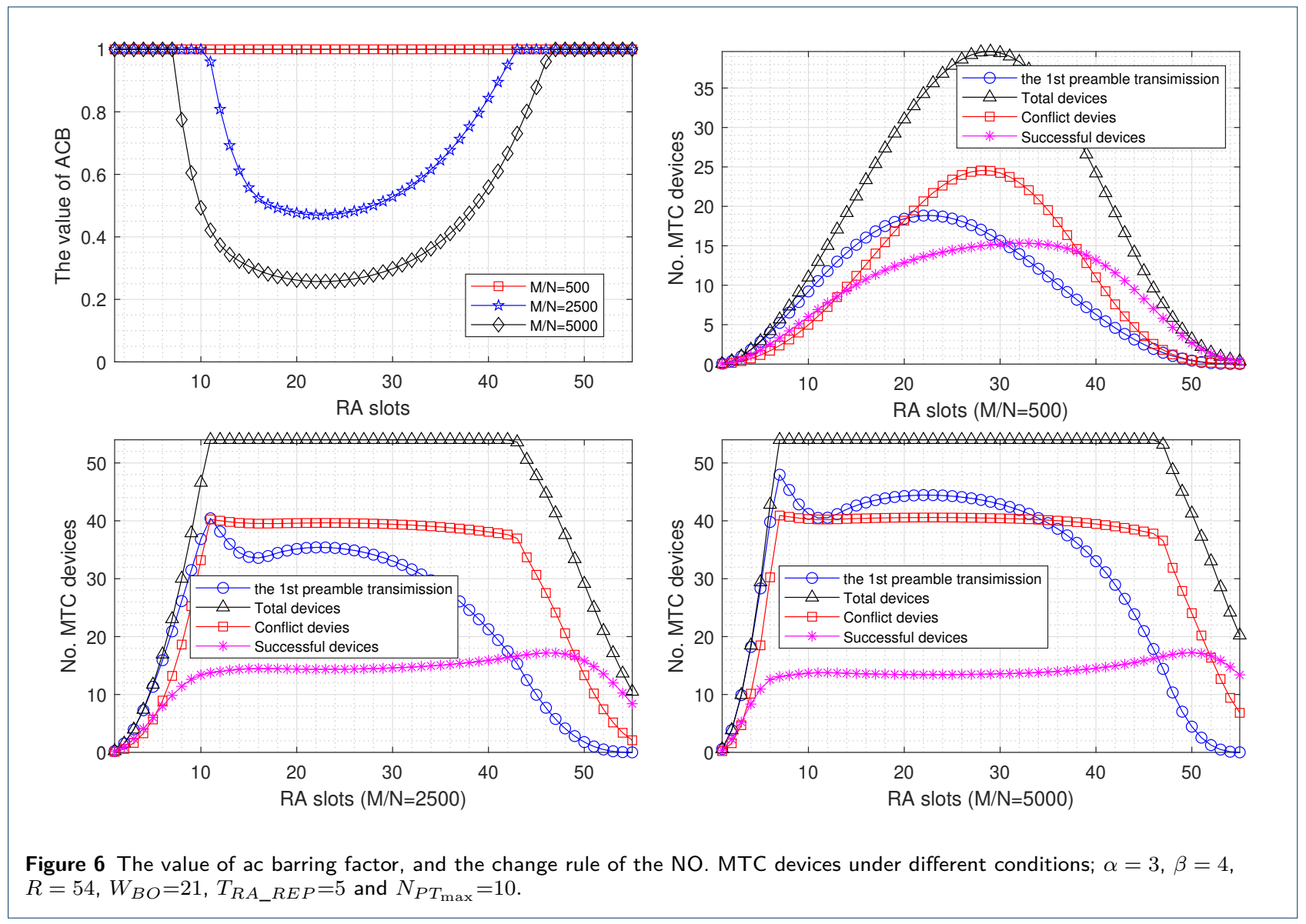


The Success Probability $\left(P_{S}\right)$ refers to the number of devices that complete the entire random access process within the maximum times of preamble transmissions divided by the total number of devices (including devices that are activated or sleep). Its definition is as follows:

$$
P_{S}=\frac{\sum_{i=1}^{I_{\max }} \sum_{n=1}^{N_{P T_{\max }}} M_{i, s}[n]}{M}
$$

\section{Collision Probability}

The Collision Probability $\left(P_{C}\right)$ is the ratio of the number that two or more MTC devices select the same preamble and transmit in the same $\mathrm{RB}$ to the total number of preambles reserved by the eNB [20]. In other words, $P_{C}$ is the radio of the total number of preambles in conflict to the total number of preambles reserved. In each random access time slot $i$, the conflicting preambles are equal to the reserved preambles $(R)$ of the eNB minus the successful preambles $\left(M_{i} e^{-\frac{M_{i}}{R}}\right)$ and the idle preambles $\left(R e^{-\frac{M_{i}}{R}}\right)[29]$. Then collision probability $P_{C}$ is:

$$
P_{C}=\frac{\sum_{i=1}^{I_{\max }}\left(R-M_{i} e^{-\frac{M_{i}}{R}}-R e^{-\frac{M_{i}}{R}}\right)}{I_{\max } R}
$$

\section{The Number of Preambles Transmission}

Let $k$ indicate the times of preamble transmission by an MTC device from the time it started transmitting an RA request to the time it completed the RA process. Cumulative distribution function (CDF), $F(k)$, is the statistical value of the number of preamble transmissions. $F(k)$ is a ratio, which refers to the ratio of the number of devices that complete the randomaccess process to the total number of completed random access processes when the number of preamble transmissions is not greater than k. $F(k)$ ranges from $0-1$. Hence, according to the reference[22], $F(k)$ is expressed as follows:

$$
F(k)=\frac{\sum_{i=1}^{I_{\max }} \sum_{m=1}^{k} M_{i, s}[m]}{\sum_{i=1}^{I_{\max }} \sum_{n=1}^{N_{P T_{\max }}} M_{i, s}[n]}
$$

\section{E. Resource Utilization}

The Resource Utilization $(R U)$ can be defined as the ratio of the total number of successful MTC devices to the total number of available preambles, and it can be given by the following equation:

$$
R U=\frac{\sum_{i=1}^{I_{\max }} \sum_{n=1}^{N_{P T_{\max }}} M_{i, s}[n]}{I_{\max } R}
$$

\subsection{Numerical Results}

Figure 7 shows the average access delay of the three mechanisms, and Figure 8 shows the CDF of access delay. From the figures, we find that the average access delay of GP grows fastest when the number of devices increases. When the number of MTC devices reaches 2000, the average access delay reaches the maximum $(>60 \mathrm{~ms})$ and remains stable. This shows that, for larger group sizes, the devices in GP may retransmit for $N_{P T_{\max }}$ times to successfully access (Figure 8). Compared with GP, PBO has an important improvement, and no matter $W_{P B O}=100$ or $W_{P B O}=240$, the access delay of $\mathrm{PBO}$ is better than GP. Especially when $W_{P B O}=240$, the access delay of PBO increases slowly when the number of devices increases. When $M / N=5000$, the average access delay of PBO $\left(W_{P B O}=240\right)$ is about $50 \mathrm{~ms}$. Compared with GP and PBO, when the number of devices in a group is small, the performance of AGO is worse than $\mathrm{PBO}\left(W_{P B O}=240\right)$, but better than GP and $\mathrm{PBO}$ $\left(W_{P B O}=100\right)$. As the number of MTC devices in the group increases (when $M / N>1000$ ), the performance is significantly better than the GP and PBO. Moreover, the delay is relatively stable and is not affect by the number of devices. For AGO, when $M / N<=700$, the access delay increases with the number of devices. However, when $M / N>700$, the access delay decreases with the increase in the number of devices, and eventually remains stable. This is because when the number of devices is small, the ACB mechanism does not work, so the delay increases as the number of devices increases. When the number of devices is large, the ACB mechanism starts to control the number of devices that access the network at the same time, so, the delay will remain stable.

According to the success probability simulation results (Figure 9), When $M / N<=500$, because the available preamble resources are greater than the number of MTC devices waiting to be accessed, the success probabilities of all methods are equal to $100 \%$. When $500<M / N<1000$, AGO, GP and PBO $\left(W_{P B O}=100\right)$ drop significantly. In comparison, the performance of $\mathrm{PBO}\left(W_{P B O}=240\right)$ is still relatively good, which can be maintained at about $100 \%$. When $1000<M / N<1700$, the performance of all mechanisms drops rapidly. In this case, $\mathrm{PBO}\left(W_{P B O}=\right.$ 
Table 1 Basic Simulation Parameters

\begin{tabular}{ccc}
\hline Notations & Definitions & Values \\
\hline \hline$M / N$ & Total number of MTC devices & $10-5000$ \\
$R$ & Available preamble resources & 54 \\
$p_{a c b}$ & AC barring factor & $0.1-0.95$ \\
$N_{P T_{\max }}$ & Maximal times of preamble transmission & 10 \\
$N_{R A R}$ & Maximal number of RAR that can be carried in a response message & 3 \\
$T_{R A R}$ & The processing delay of the eNB & 2 \\
$W_{R A R}$ & Size of the random-access response window & 5 \\
$N_{A C K}$ & Maximal number of MTC devices that can be acknowledged within the RAR window & $N_{R A R} \times W_{R A R}$ \\
$T_{R A-R E P}$ & Interval between two consecutive random-access slots & 5 \\
$W_{B O}$ & Size of back-off window & $B I+1$ \\
$p_{n}$ & Detection probability & $p_{n}=1-e^{-n}$ \\
$B I$ & Back-off indicator & 20 \\
\hline$T_{M S G}$ & The average time for the device to successfully transmit information & 11 \\
\hline
\end{tabular}

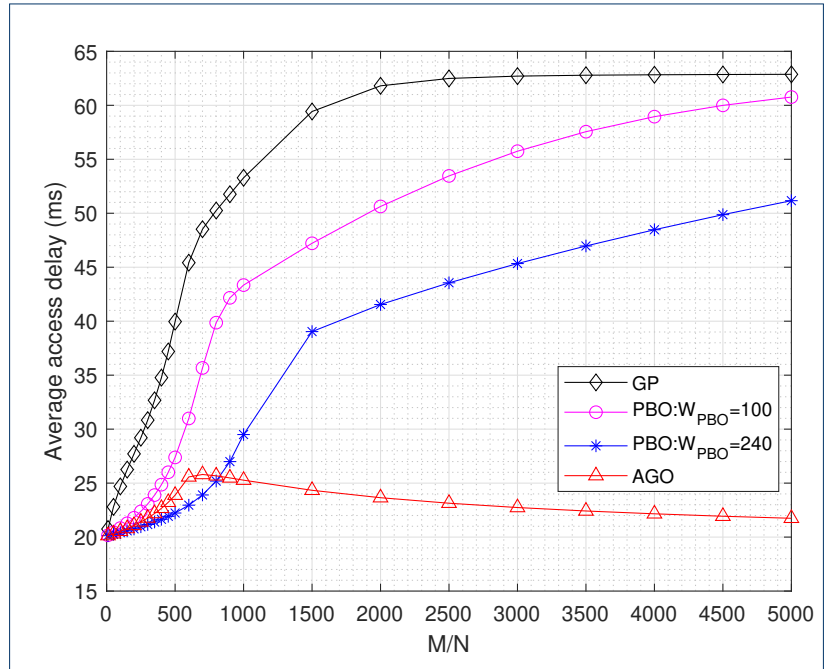

Figure 7 Average access delay for the considered methods.

240) performs better than AGO. When the number of MTC devices is large (e.g., more than 2000), the GP and PBO drop significantly. In contrast, the performance degradation of the AGO is relatively flat. When $M / N=5000$, the success probability can still be maintained at more than $15 \%$. This means that AGO is more suitable for the scenario of large-scale MTC devices.

As shown in Figure 10, AGO has effectively alleviated the network congestion problem. As the number of MTC devices increases, the collision probability of AGO increases slightly, but the increase rate is slower. Finally, the collision probability is controlled at about $20 \%$. For the GP and the PBO $\left(W_{P B O}=100\right)$, as the number of MTC devices increases, the collision probability increases rapidly. When $M / N=500$ to 1500 , the collision probability increases rapidly from $20 \%$ to about $60 \%$. Then the collision probability tends to be stable. When $M / N>=4000$, the collision probability is over $70 \%$, and close to $80 \%$. PBO $\left(W_{P B O}=240\right)$ performs better than GP and $\mathrm{PBO}\left(W_{P B O}=100\right)$,

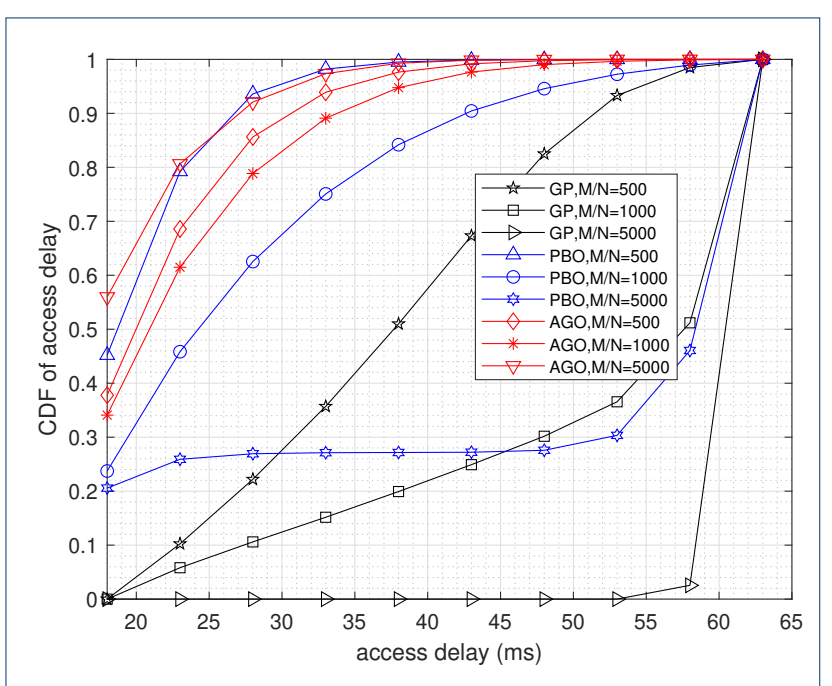

Figure 8 The CDF of average access delay for the considered methods.

but when $M / N>2000$, the collision probability exceeds $70 \%$ too. In summary, when the number of MTC devices is large (e.g., more than 3500), the collision probabilities of GP and PBO are close to $80 \%$. However, AGO always controls the collision probability below $30 \%$ through the ACB mechanism.

Figure 11 shown the average number of preamble transmission, and Figure 12 shows the CDF of the preamble transmissions. In general, the performance of PBO $\left(W_{P B O}=240\right)$ is better than GP, and the performance of AGO is better than both of them. In GP, in the case of $M / N=500$, when the number of preamble transmission exceeds 5 , the probability of success can reach $50 \%$. And more than 7 times, the probability of success can exceed $80 \%$ (Figure 12). For PBO, in the case of $M / N=500$, it performs best. But when the number of MTC device is large (e.g., 1000,5000), its performance drops. Especially when $M / N=5000$, the average number of preamble transmission exceeds 7. This means that more retransmissions are required 


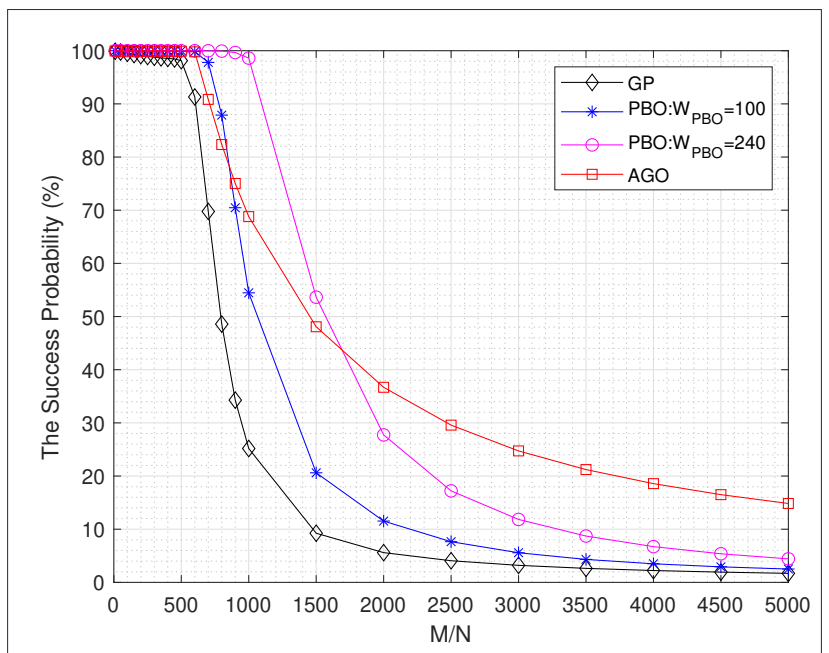

Figure 9 The success probability for the considered methods.

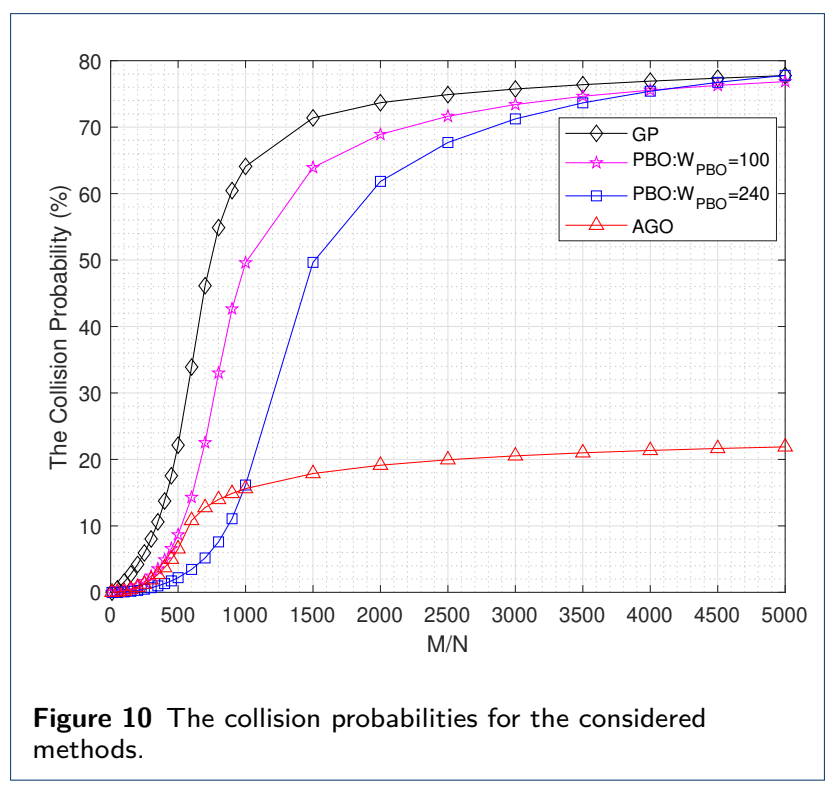

to complete the access process. In comparison, AGO has little change in performance when $M / N=500$ and $M / N=1000$. When the number of preamble transmissions exceeds 3 times, the success rate of AGO can reach $80 \%$ (Figure 12). When the number of MTC device reach 5000, due to the $\mathrm{ACB}$ mechanism, the performance is better than when $M / N=500$ or 1000. In summary, when the number of devices in the group is consistent, AGO significantly reduces the times of preamble transmissions compared to other methods. From the perspective of energy consumption, our proposed method reduces the number of retransmissions, thereby reducing energy consumption, so it is more conducive to the communication of MTC devices.

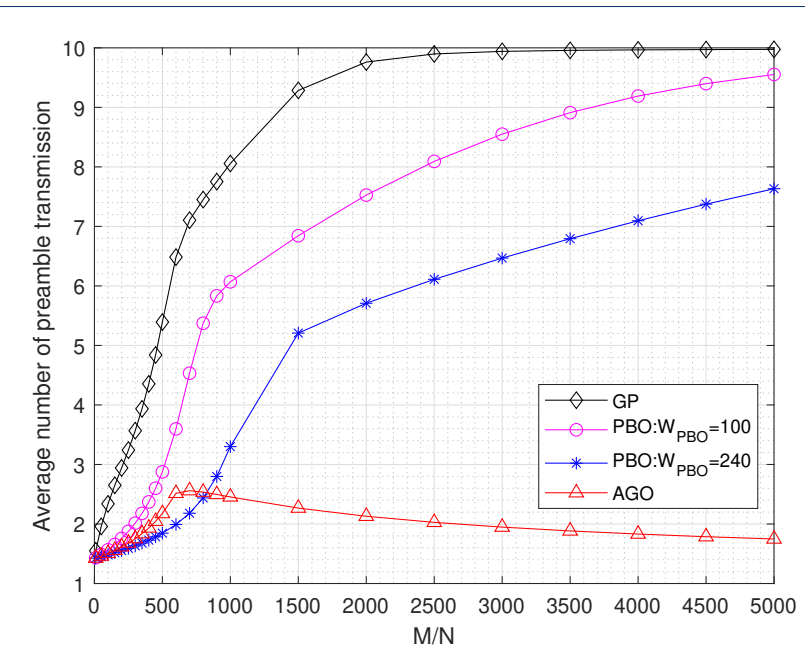

Figure 11 The average number of preamble transmission for the considered methods.

As shown in Figure 13, from the perspective of resource utilization, when the number of MTC devices is relatively small (e.g., $M / N<700$ ), the RU rate of GP is better than PBO. When the number of MTC devices is large (e.g., $M / N>700$ ), PBO behaves better than GP. For PBO, when the number of MTC devices is small (e.g., $M / N<1000)$, $\mathrm{PBO}\left(W_{P B O}=100\right)$ achieve a better performance, in contrast, when the number of MTC devices is large (e.g., $M / N>1000$ ), PBO $\left(W_{P B O}=240\right)$ performs better. Combining the previous conclusions, we find that $\mathrm{PBO}\left(W_{P B O}=240\right)$ performs better in large-scale device scenarios. However, with the increase of MTC devices, the RU decreases significantly, and finally the RU is less than $5 \%$. Compared with GP and PBO, AGO achieves higher RU (when $M / N=1000, R U=23.16 \%$ ). AGO does not decrease as the number of MTC devices increases, especially when the number of MTC devices is large (e.g., 5000), AGO remains at about $25 \%$. This shows that the ACB mechanism controls the number of devices well, thereby improving RU.

Figure 14 uses the access success probability as an example to show the impact of different ac barring factors on AGO. As shown in the figure, there are four methods, AGO with the dynamic ac barring factor and AGO with the fixed ac barring factor $(0.3,0.6,0.9)$ respectively. When $p_{a c b}$ is small (e.g., 0.3 or 0.6$)$, even when there are relatively few MTC devices (e.g., 500), the overall success probability is low because the number of devices that pass the ACB mechanism is relatively small. When $p_{a c b}$ is large (e.g., 0.9 ), when the number of MTC devices is relatively small (e.g., <1000), the performance is better. However, when the number of MTC devices increases, the 


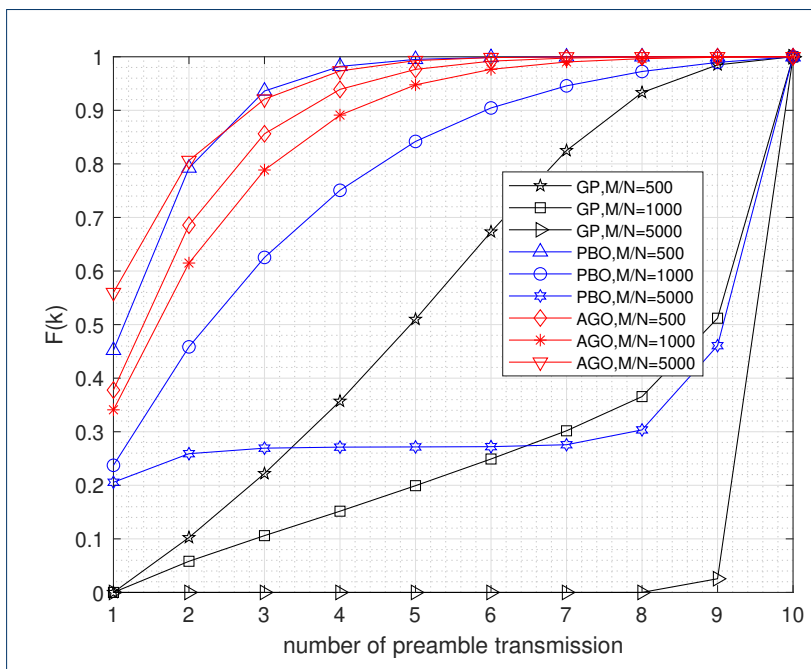

Figure 12 The CDF of average number of preamble transmissions for the considered methods.

performance drops sharply. When $M / N=5000$, the access success probability drops to between $2 \%$ and $3 \%$. AGO, which uses the dynamic ACB parameter adjustment method, achieves the best performance because it can adaptively adjust the parameters according to the access load.

\section{Conclusions}

In this paper, we have proposed an ACB-based group paging overload control method for massive MTC accesses in LTE network. Instead of all MTC devices in the group can access the random-access process, only MTC devices passed the ACB mechanism can access the random-access process. In this paper, we first scatter the MTC devices over a GP interval reduce access conflicts. And then we assume that the newly arrived MTC devices obey the beta distribution, and calculate the number of newly arrived devices. We calculate the number of devices needed to access the network in the current time slot according to the number of newly arrived devices and the devices in the previous time slot that failed to access. Based on this, AGO can adjusted the ACB parameters dynamically. AGO has been evaluated for a relatively large number of MTC devices (5000 MTC devices). Compared with GP and PBO mechanisms, AGO achieves many improvements, in term of success probability, collision probability, resource utilization rate and average access delay, etc. Beside the success probability, average access delay improvements, AGO controls the collision probability very well. When the number of MTC devices is large (5000 MTC devices), the collision probability can also be controlled at about $20 \%$. In addition, AGO also achieved good results in terms of average number of

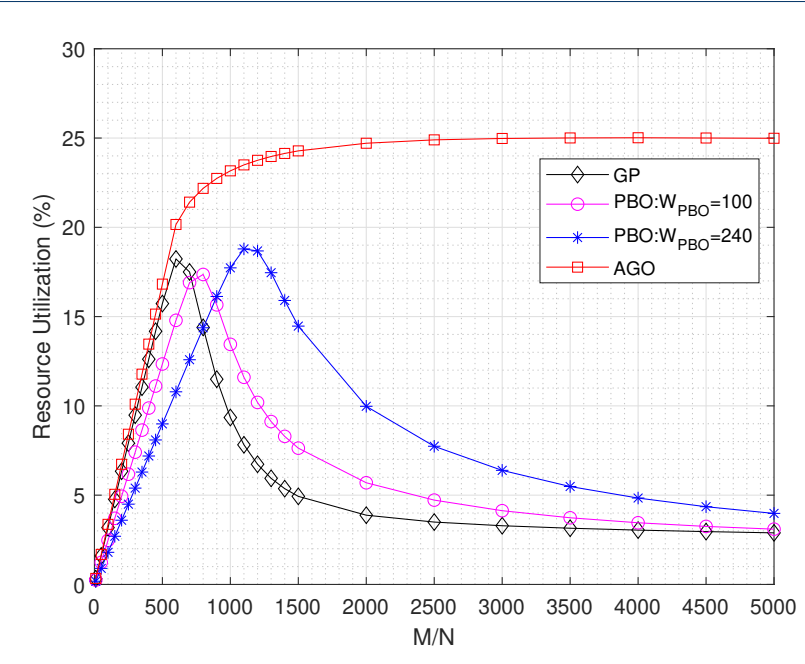

Figure 13 The resource utilization for the considered methods

preamble transmissions. Regardless of the number of MTC devices, AGO can achieve a success probability of about $90 \%$ under the preamble transmission of about 4 times. This shows that the number of devices in the group has little effect on AGO. Therefore, AGO is more suitable for massive MTC device application scenarios.

\section{Abbreviations}

ACB: Access Class Barring; GP: Group Paging; PBO: Pre-BackOff; AGO: ACB-based Group Paging Overload Control Method; loT: Internet of Thing; MTC: Machine Type Communication; M2M: Machine to Machine Communication; LTE: Long Term Evolution; PUSCH: Physical Uplink Shared Channel; GID: Group ID; RAR: Random Access Response; RA: Random Access; RACH: Random Access Channel; EAB: Extended Access Barring; SIB: System Information Block; RU: Resource Utilization; CDF: Cumulative Distribution Function.

\section{Acknowledgements}

Not applicable.

\section{Author's contributions}

Wang Cong and Wei Chengqiang conceived the idea and wrote the paper; Li Ning analyzed the simulation results; Ma Wenfeng and Tian Hui performed the experiments. All of the authors participated in the project, and they read and approved the final manuscript.

\section{Funding}

This work was supported by the National Natural Science Foundation of China under grant 61771486

\section{Competing interests}

The authors declare that they have no competing interests.

\section{Author details}

${ }^{1}$ College of Field Engineering, Army Engineering University of PLA, 210007

Nanjing, China. ${ }^{2}$ College of Communications Engineering, Army

Engineering University of PLA, 210007 Nanjing, China.

\section{References}

1. Andreev, S., Galinina, O., Pyattaev, A.: Understanding the iot connectivity landscape: A contemporary $\mathrm{m} 2 \mathrm{~m}$ radio technology roadmap. IEEE Communications Magazine 53, 32-40 (2015)

2. Lawton, G.: Machine-to-machine technology gears up for growth. Computer 37, 12-15 (2014) 


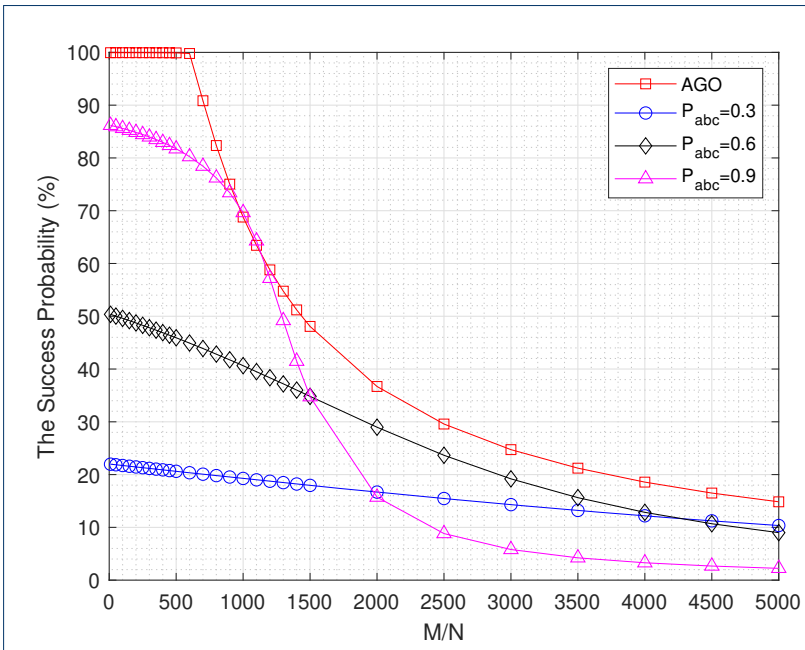

Figure 14 Comparison of access success probability for different ac barring factor

3. Kartsakli, E., Lalos, A., Antonopoulos, A.: A survey on $\mathrm{m} 2 \mathrm{~m}$ systems for mhealth: A wireless communications perspective. Sensors 14, 18009-18052 (2014)

4. Chen, M., Wan, J., Gonzalez, S., Liao, X., Leung, V.C.M.: A survey of recent developments in home $\mathrm{m} 2 \mathrm{~m}$ networks. IEEE Communications Surveys \& Tutorials 16, 98-114 (2015)

5. 3GPP: 3GPP RAN2 71: Pull Based RAN Overload Control. Madrid Spain (2010.08). 3GPP

6. 3GPP: 3GPP TR 23.898: Access Class Barring and Overload Protection; (Release 7). (2005.03). 3GPP

7. Hussain, F., Anpalagan, A., Vannithamby, R.: Medium access control techniques in $\mathrm{m} 2 \mathrm{~m}$ communication: Survey and critical review. Transactions on Emerging Telecommunications Technologies 28 (2014)

8. Sui, N., Xu, Y., Wang, C., Xie, W.: Performance analysis of a novel hybrid s-aloha/tdma protocol for beta distributed massive mtc access. Sensors 17 (2017)

9. Arouk, O., Ksentini, A., Hadjadj, A.Y., Taleb, T.: On improving the group paging method for machine-type-communications. In: IEEE (ed.) the 2014 IEEE International Conference on Communications, June 2014, pp. 484-489 (2014)

10. Harwahyu, R., Cheng, R., Sari, R.F.: Consecutive group paging for Ite networks supporting machine-type communications services. In: IEEE (ed.) the 2013 IEEE 24th International Symposium on Personal Indoor and Mobile Radio Communications (PIMRC) (2013)

11. Arouk, O., Ksentini, A., Taleb, T.: Group paging-based energy saving for massive mtc accesses in Ite and beyond networks. IEEE JOURNAL ON SELECTED AREAS IN COMMUNICATIONS 34, 1086-1102 (2016)

12. Jiang, W., X, W., Deng, T.: Performance analysis of a pre-backoff based random access scheme for machine-type communications. In: the 2014 International Conference on Intelligent Green Building and Smart Grid (IGBSG 2014), pp. 1-4 (2014). Taipei, Taiwan

13. Harwahyu, R., Wang, X., Sari, R., Cheng, R.G.: Analysis of group paging with pre-backoff. EURASIP Journal on Wireless Communications and Networking 34 (2015)

14. Chen, J., Lin, Y., Cheng, R.: A delayed random access speed-up scheme for group paging in machine-type communications. In: the 2015 IEEE International Conference on Communications, pp. 623-627 (2015). Taipei, Taiwan

15. Ksentini, A., Hadjadj-Aou, Y., Taleb, T.: Cellular-based machine-to-machine: Overload control. IEEE Network 26, 54-60 (2012)

16. He, H., Du, Q., Song, H.: Traffic-aware acb scheme for massive access in machine-to-machine networks. In: the 2015 IEEE International Conference on Communications (ICC), pp. 617-622 (2015)

17. Duan, S., Shah-Mansouri, V., Wong, V.W.S.: Dynamic access class barring for $\mathrm{m} 2 \mathrm{~m}$ communications in Ite networks. In: the 2013 IEEE Globecom Workshops (GC Wkshps), pp. 9-13 (2013)

18. Duan, S., Shah-Mansouri, V., Wang, Z.: D-acb: Adaptive congestion control algorithm for bursty $\mathrm{m} 2 \mathrm{~m}$ traffic in Ite networks. IEEE Transactions on Vehicular Technology 65, 9847-9861 (2016)

19. Zangar, N., Gharbi, S., Abdennebi, M.: Service differentiation strategy based on macb factor for $\mathrm{m} 2 \mathrm{~m}$ communications in Ite-a networks. In: the 2016 13th IEEE Consumer Communications \& Networking Conference, pp. 9-12 (2016)

20. 3GPP: 3GPP TR 37.868: Study on RAN Improvements for Machine-Type Communications. Sophia-Antipolis Cedex, France (2011.08). 3GPP

21. Phuyal, U., Koc, A., Fong, M.H.: Controlling access overload and signaling congestion in $\mathrm{m} 2 \mathrm{~m}$ networks. In: the 2012 Conference Record of the Forty Sixth Asilomar Conference on Signals, Systems and Computers (ASILOMAR), pp. 4-7 (2012)

22. Wei, C.H., Cheng, R.G., Tsao, S.L.: Performance analysis of group paging for machine-type communications in Ite networks. IEEE Transactions on Vehicular Technology 62, 3371-3382 (2013)

23. Arouk, O., Ksentini, A., Taleby, T.: Group paging optimization for machine-type communications. In: the 2015 IEEE International Conference on Communications (2015)

24. Phuyal, U., Koc, A.T., MH, P., Vannithamby, R.: Controlling access overload and signalling congestion in $\mathrm{m} 2 \mathrm{~m}$ networks. In: the Forty Sixth Asilomar Conference on Signals, Systems and Computers, pp. 591-595 (2012)

25. Jang, H.S., Jung, B.C., Sung, D.: Dynamic access control with resource limitation for group paging-based cellular iot systems $\mathbf{5}$, 5065-5075 (2018)

26. Cao, C., Li, N., Wang, C., Xie, W.: Dynamic allocation of rach resource for delay-sensitive devices in $\mathrm{m} 2 \mathrm{~m}$ communications. In: the 2016 5th International Conference on Computer Science and Network Technology (2016)

27. Oh, C.-Y., Hwang, D., Lee, T.-J.: Joint access control and resource allocation for concurrent and massive access of $\mathrm{m} 2 \mathrm{~m}$ devices. IEEE Transactions on Wireless Communications 14, 1-1 (2015) doi:10.1109/TWC.2015.2417873

28. Gupta, A.K., Nadarajah, S.: Handbook of Beta Distribution and Its Applications. CRC Press, Boca Raton, Florida, USA (2004)

29. Wei, C.H., Cheng, R.G., Tsao, S.L.: Modeling and estimation of oneshot random access for finite-user multichannel slotted aloha systems. IEEE Communications Letters 16, 1196-1199 (2012) 
Figures

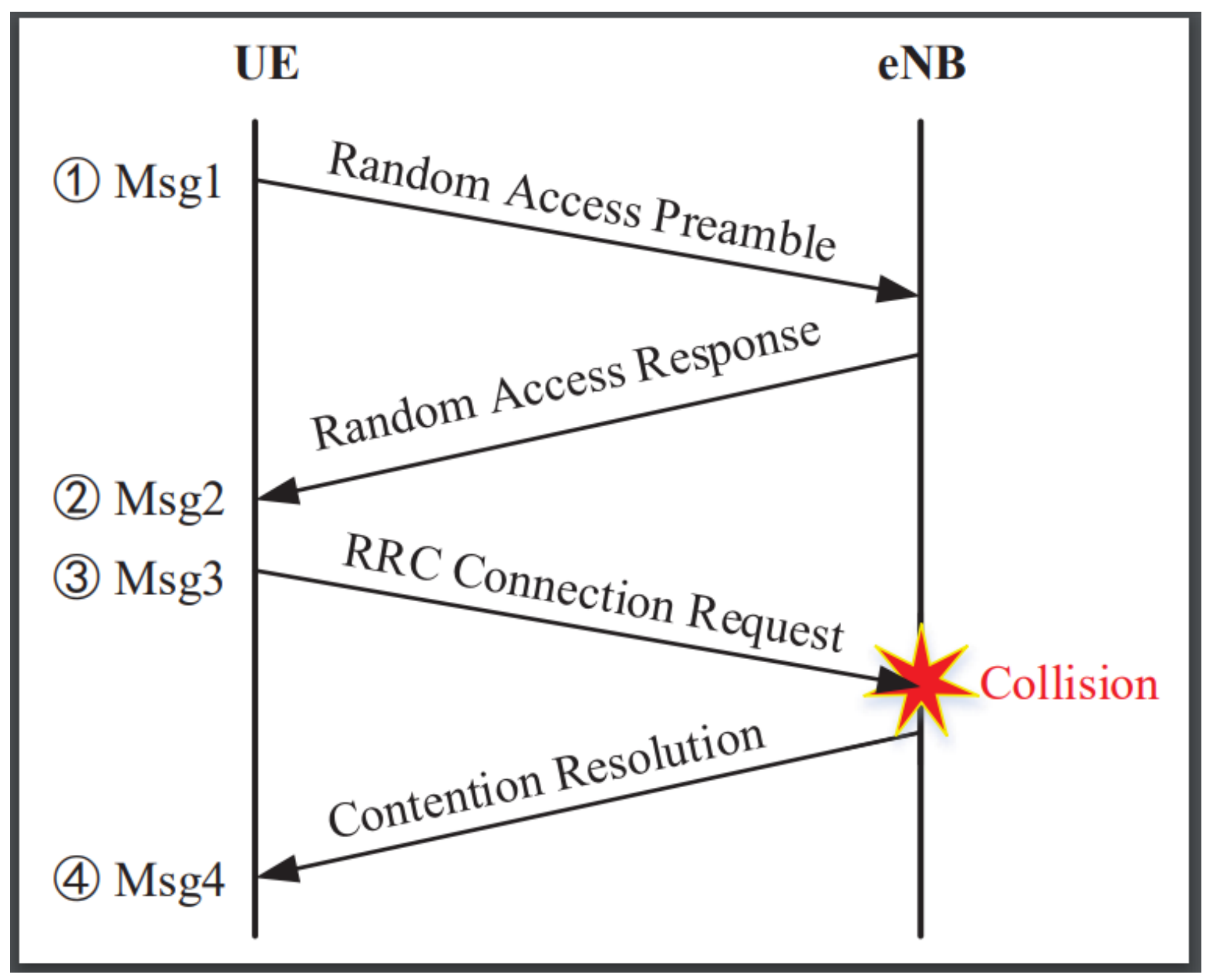

Figure 1

Random access procedure 


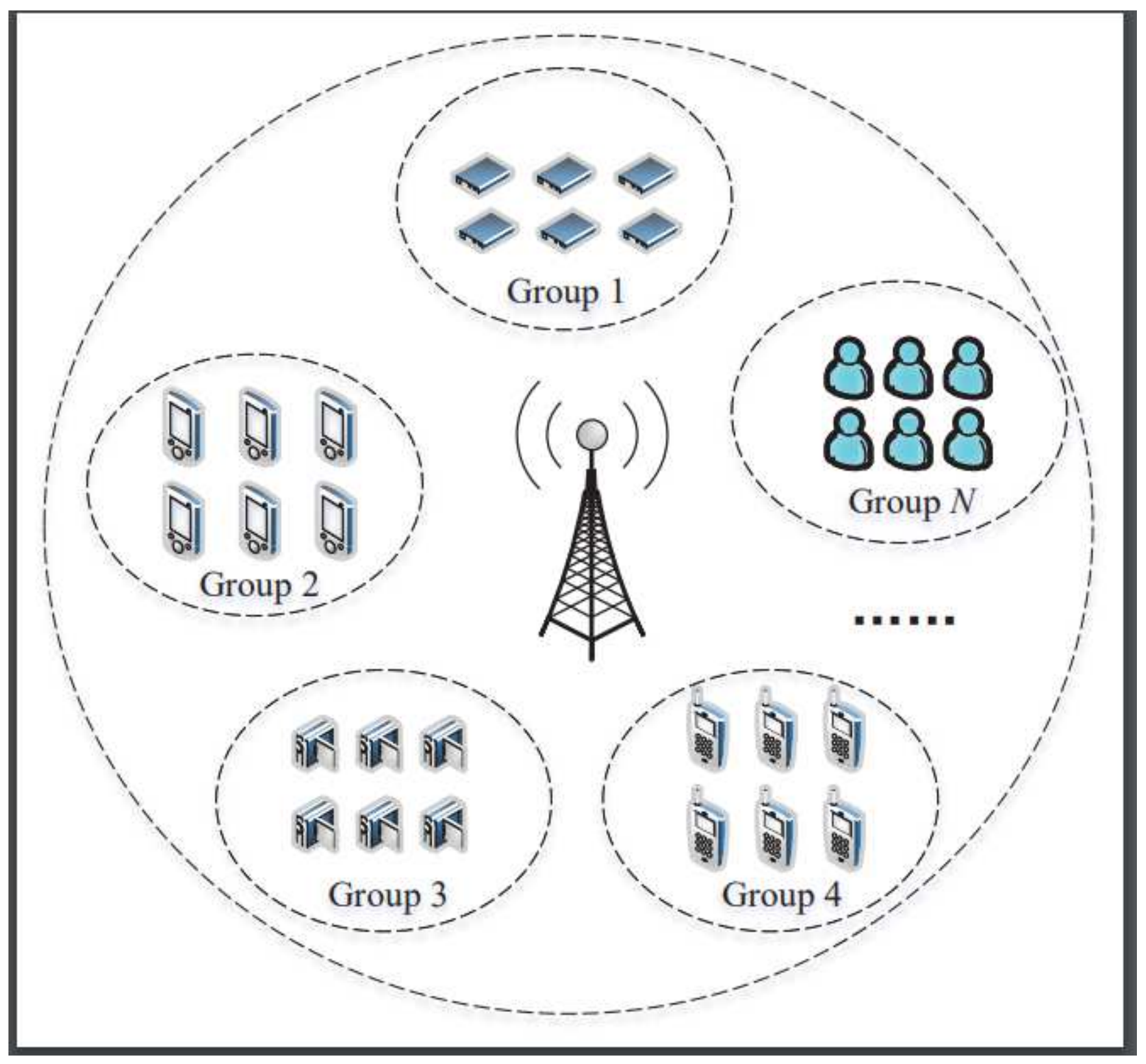

Figure 2

System Model 

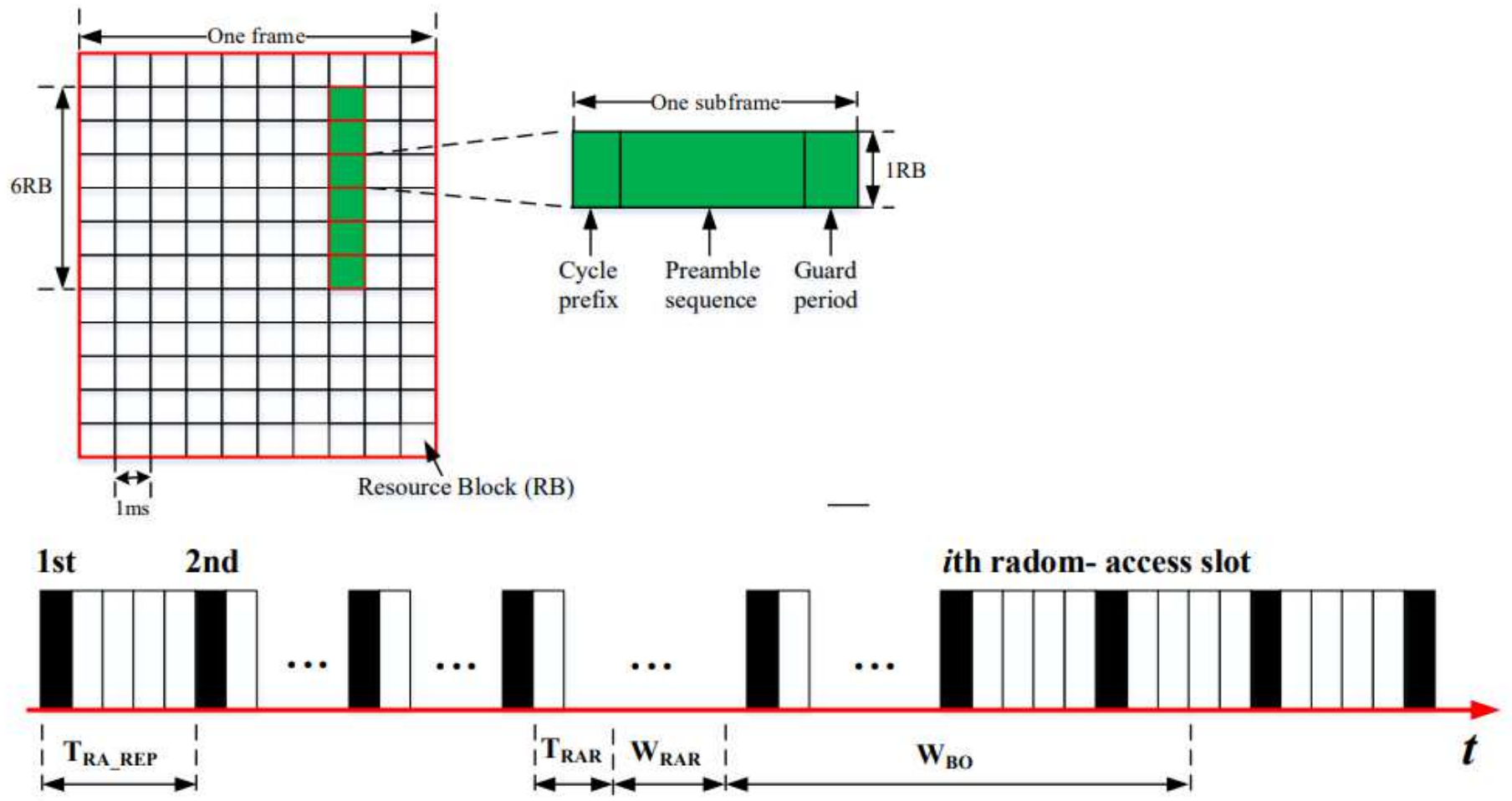

$\stackrel{\uparrow}{t=0}$

Figure 3

Random access time slot and resource allocation 


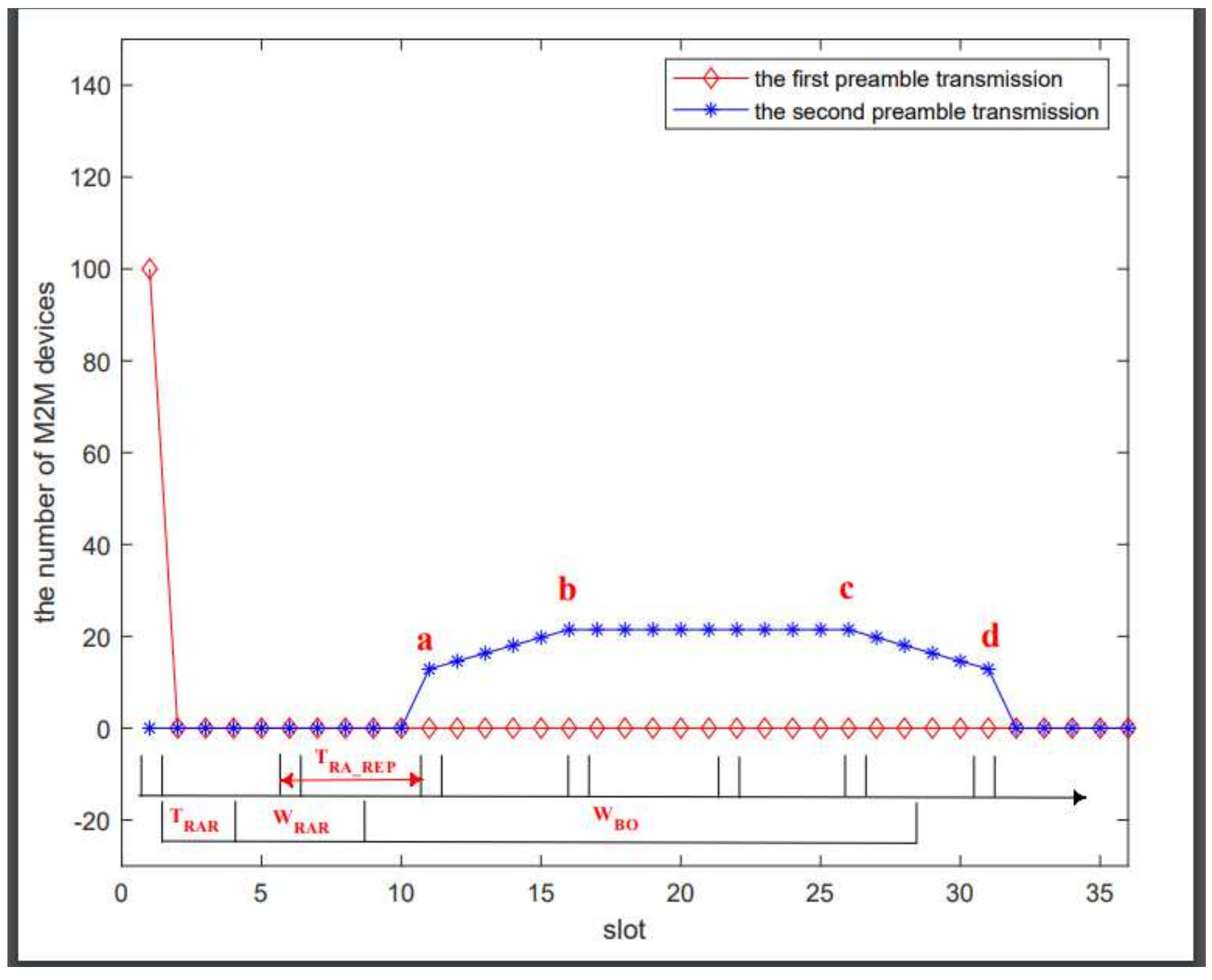

Figure 4

Number of MTC devices at each RA slot for the first and second preamble transmission for $\mathrm{R}=54$ and $\mathrm{M} / \mathrm{N}=100[11]$ 


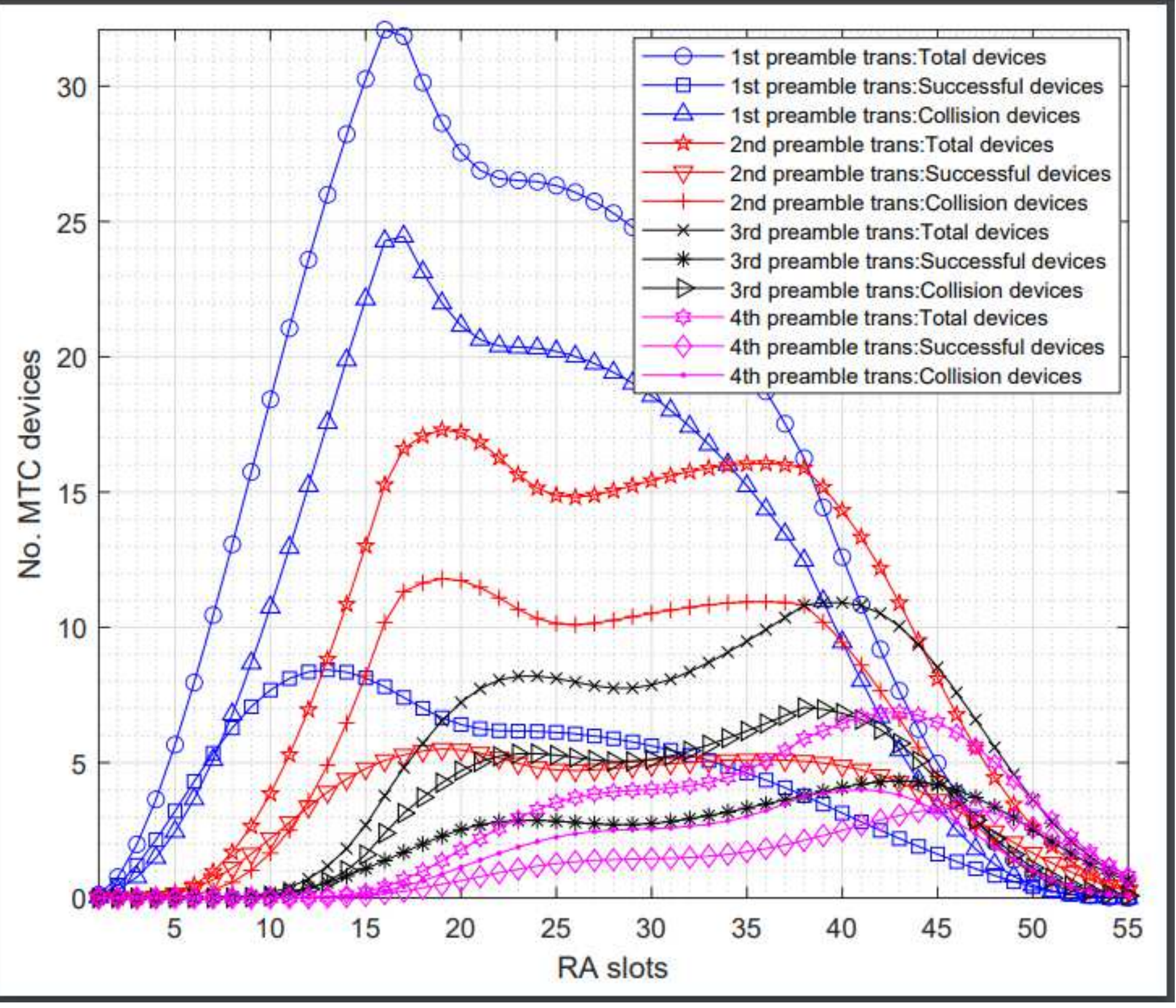

\section{Figure 5}

The number of the total devices, the successful devices and the collision devices for each preamble transmission in each random-access slot; $M / N=1000, P \operatorname{Tmax}=10, a=3, \beta=4$. 

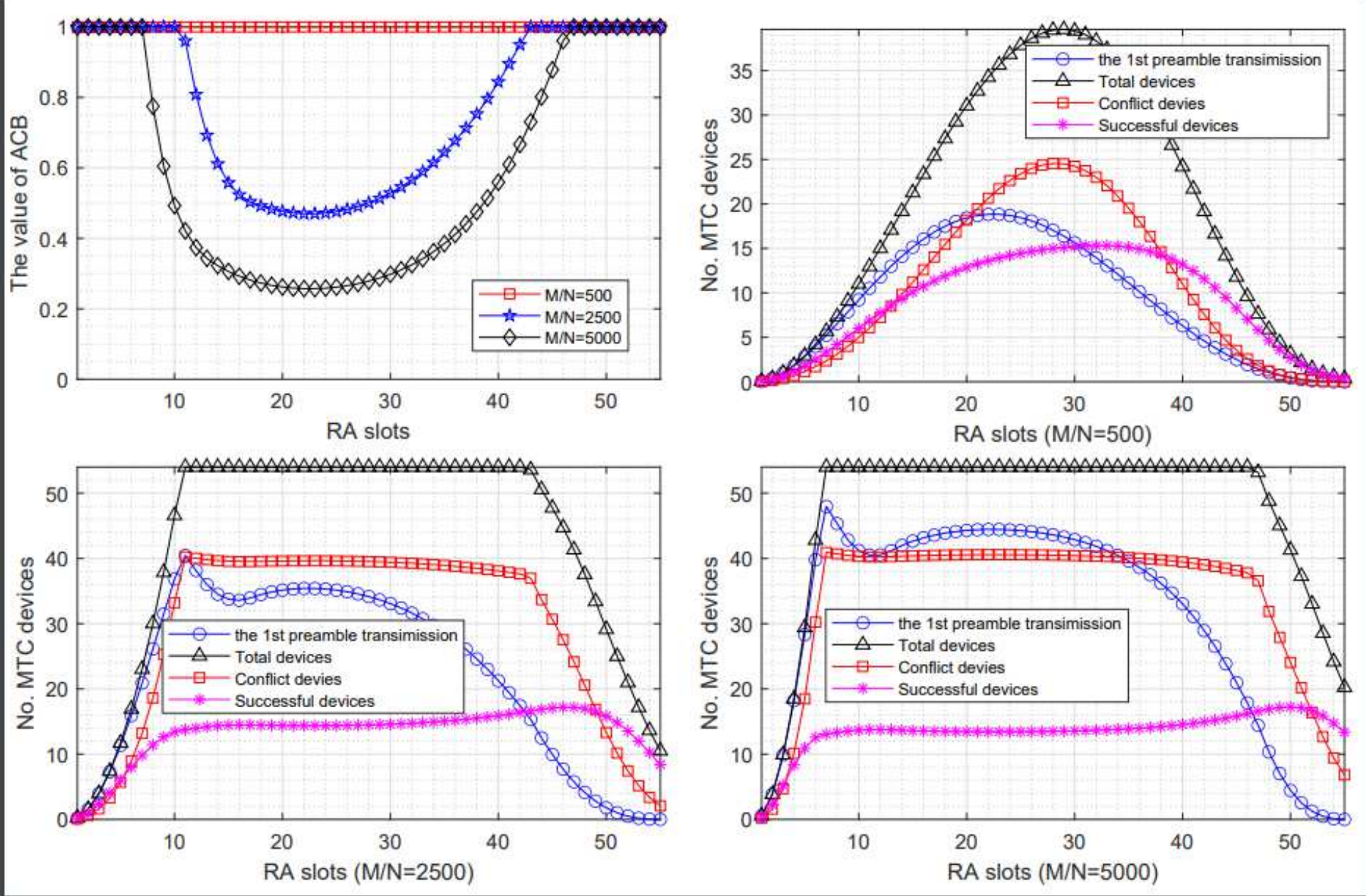

Figure 6

The value of ac barring factor, and the change rule of the NO. MTC devices under different conditions; $a=$ $3, \beta=4, R=54, W B O=21$, TRA_REP $=5$ and NP Tmax $=10$. 


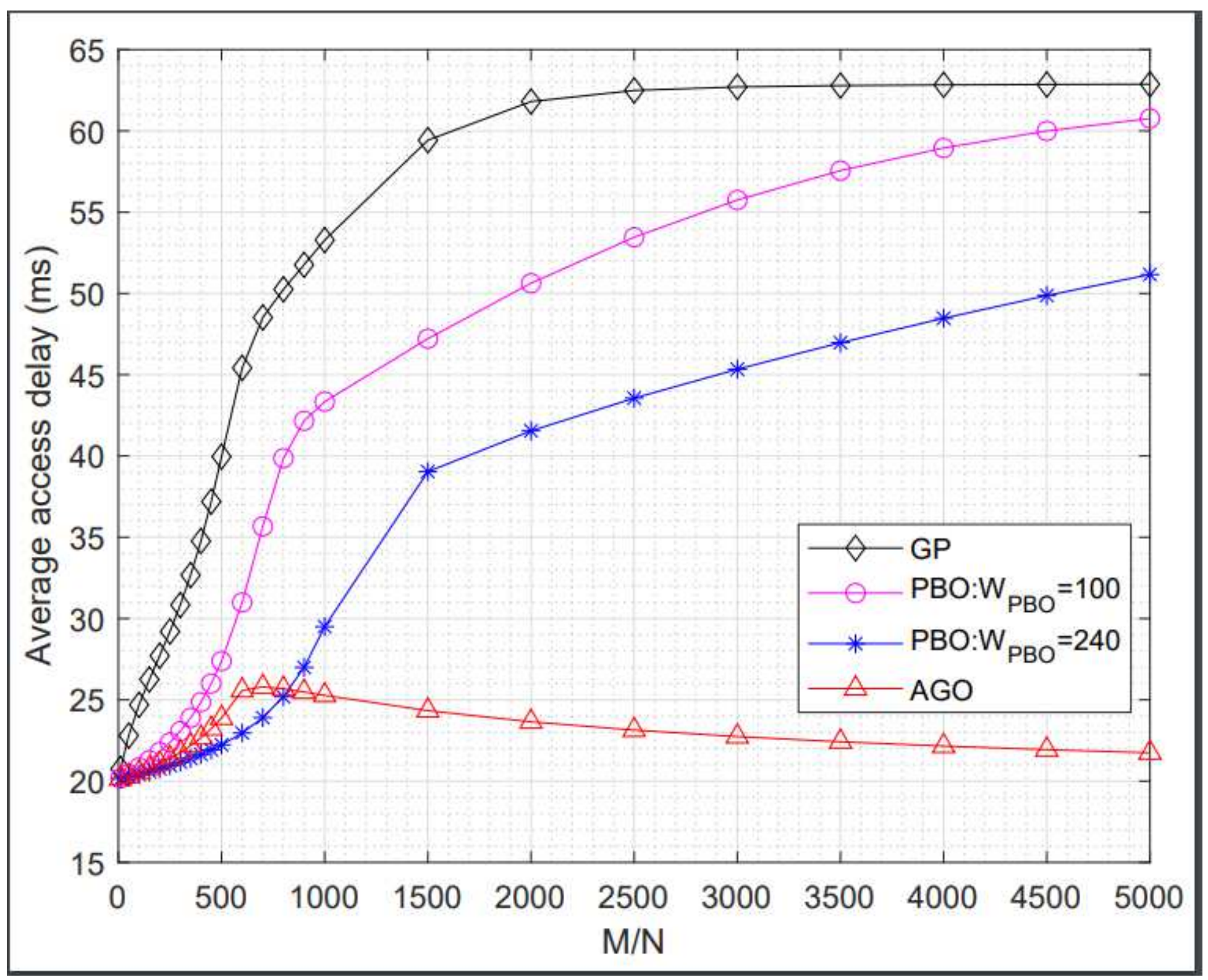

Figure 7

Average access delay for the considered methods. 


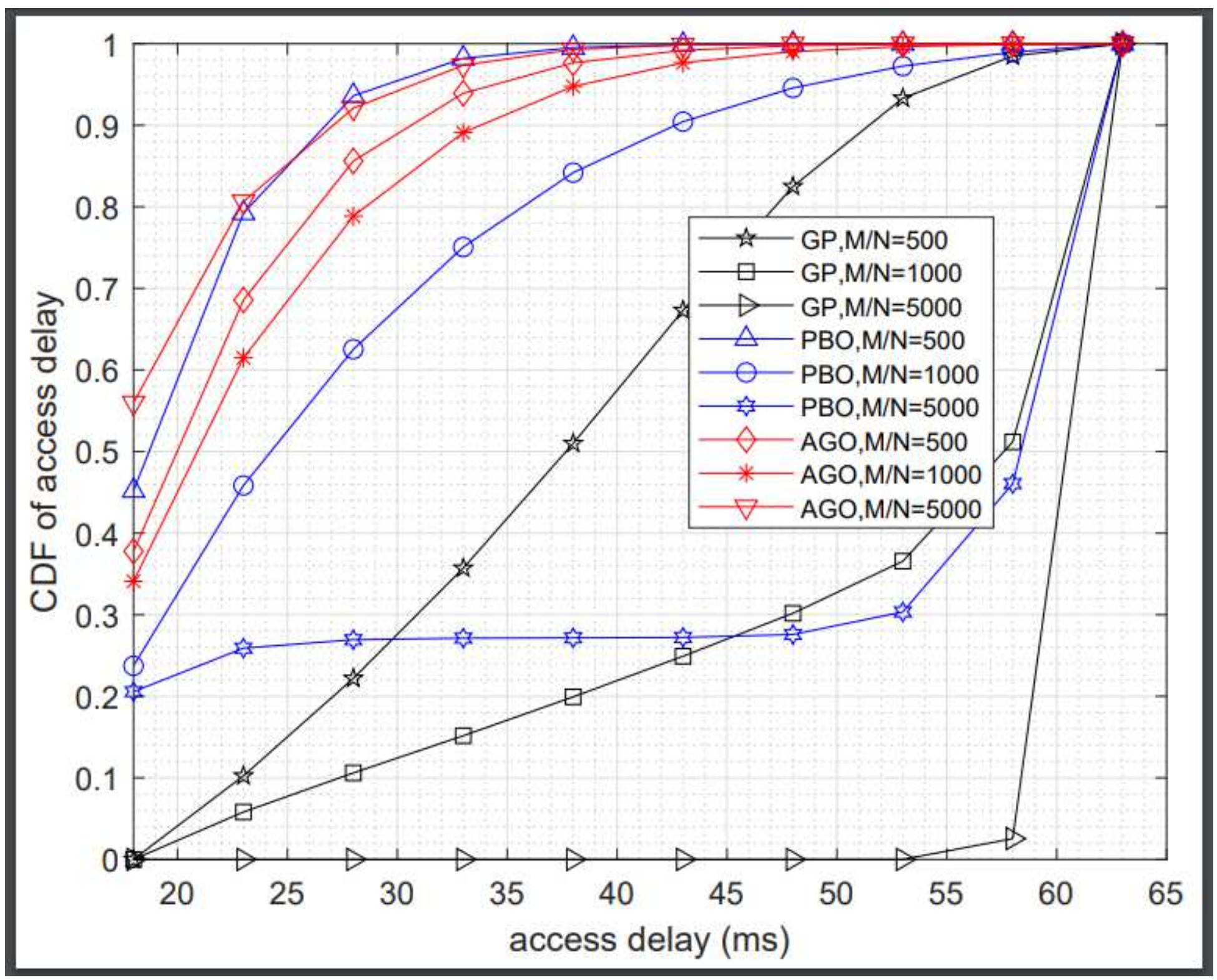

Figure 8

The CDF of average access delay for the considered methods. 


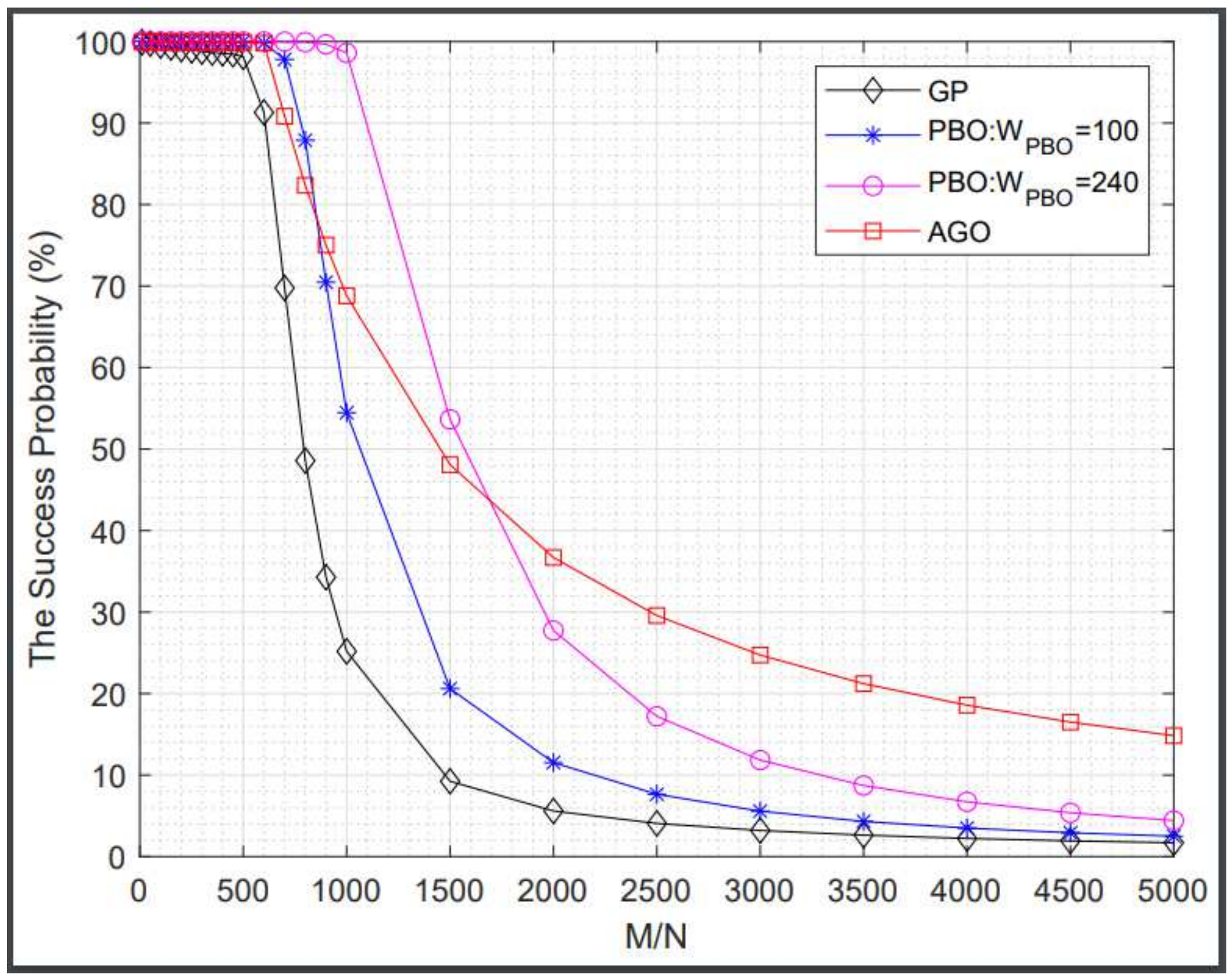

Figure 9

The success probability for the considered methods. 


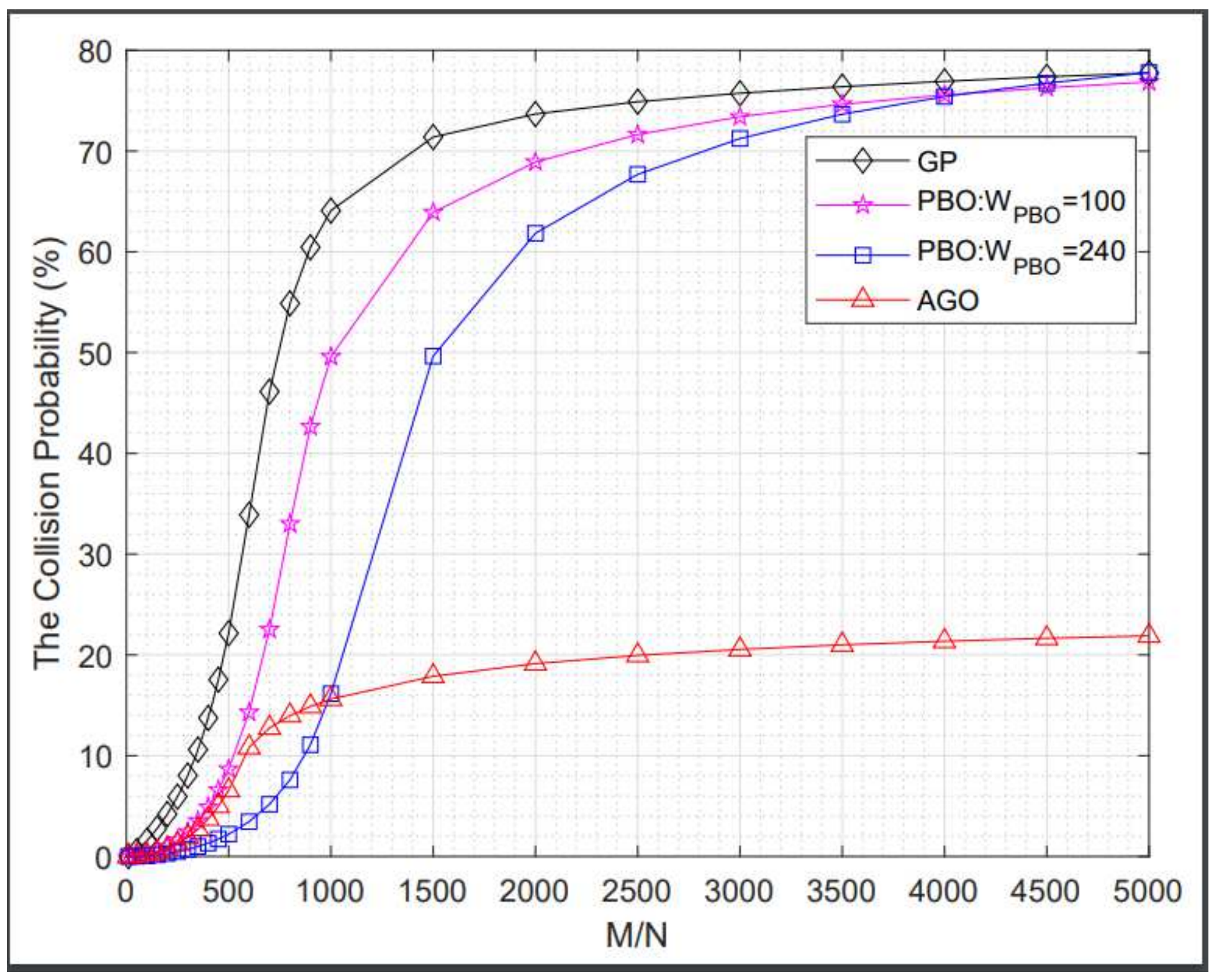

Figure 10

The collision probabilities for the considered methods. 


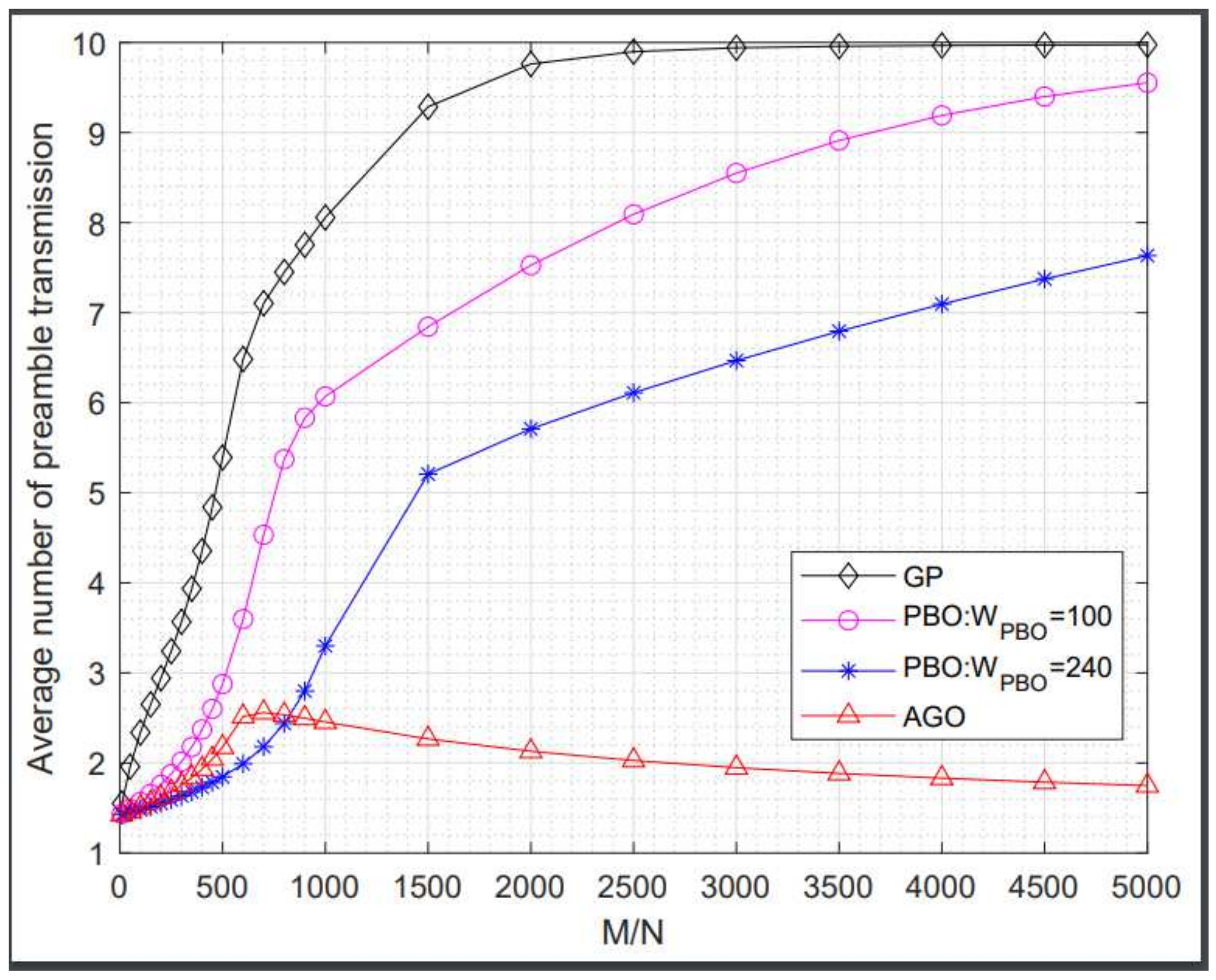

Figure 11

The average number of preamble transmission for the considered methods. 


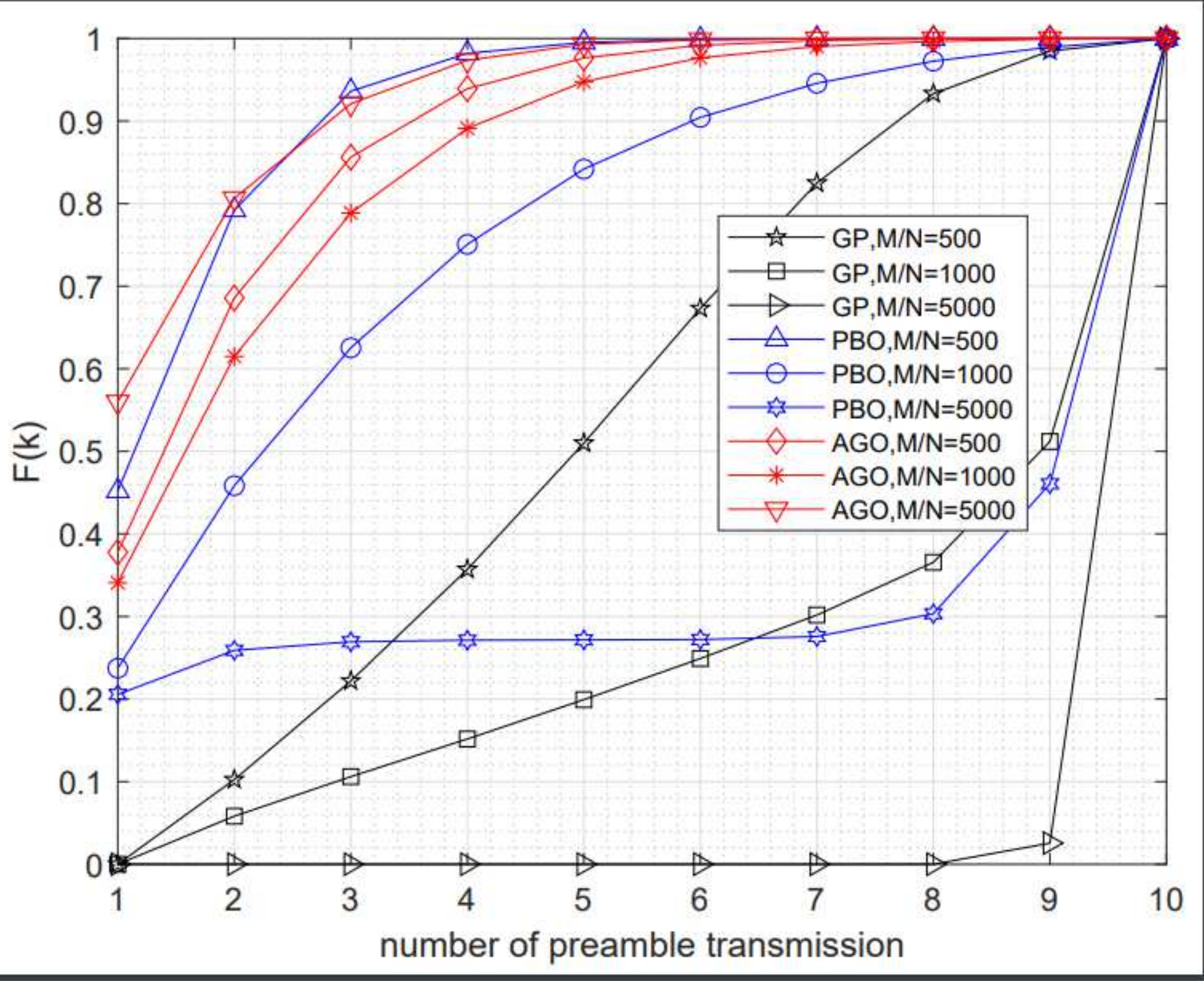

Figure 12

The CDF of average number of preamble transmissions for the considered methods. 


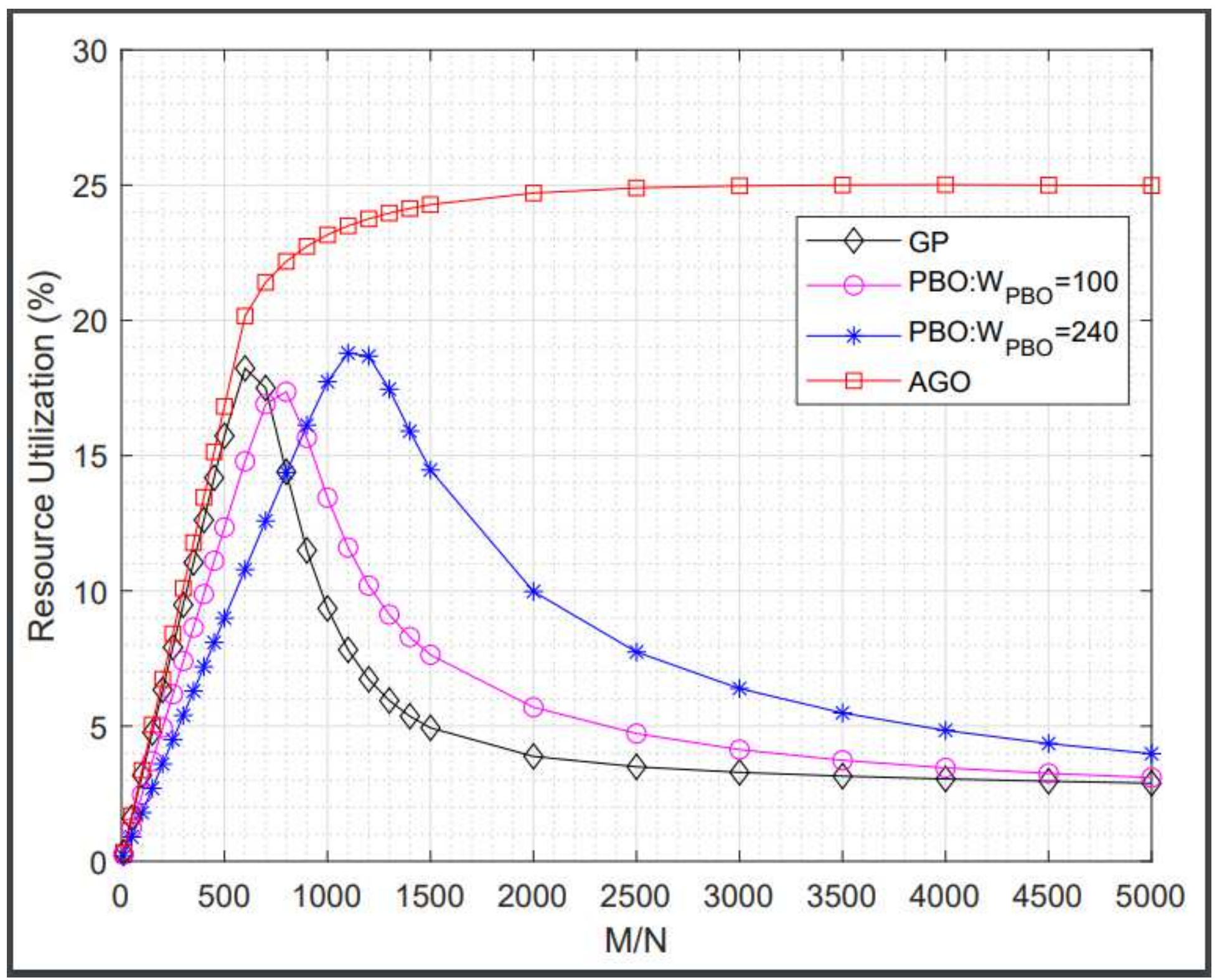

Figure 13

The resource utilization for the considered methods 




Figure 14

Comparison of access success probability for different ac barring factor 